The User's View for the Future of LAMPF, 1989:

Reports from the Pion

Physics Working Group

Compiled by

G. R. Burleson*

D. J. Ernst ${ }^{* *}$

"Guest Scientist at Los Alamos. Physics Department, New Mexico State Unizersity, Las Cruces, NM 88003.

* Guest Scientist at Los Alamos. Physics Department, Texas AEM

University, College Station, TX 77843. 


\title{
THE USER'S VIEW FOR THE \\ FUTURE OF LAMPF, 1989: \\ REPORTS FROM THE \\ PION PHYSICS WORKING GROUP
}

\author{
Compiled by
}

G. R. Burleson and D. J. Ernst

\begin{abstract}
This report contains a collection of papers on pion-nucleus interactions that were written as part of the long-range planning process of LAMPF that took place in spring, 1989. These papers served as the basis of the pion portion of a report to the Nuclear Science Advisory Committee (NSAC) for its Long Range Plan. They were read and discussed in some detail by the pion physics community and represent the views of the present and the future of pion physics by the authors and to a great extent by the pion physics community as a whole.
\end{abstract}




\section{CONTENTS}

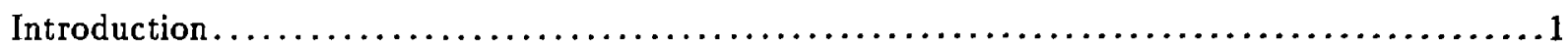

Pion-Nucleon Resonances (M. E. Sadler, T. Goldman, and E. R. Kinney)................4

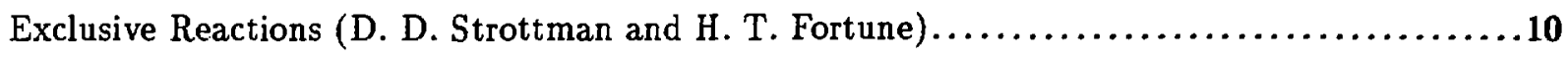
Pion Propagation in Nuclei (J. L. Matthews, R. P. Redwine, and W. R. Gibbs)............14 Meson Production (W. R. Gibbs, J.-C. Peng, C. S. Mishra, and D. Isenhower).............18 $\left(\pi^{+}, K^{+}\right)$Physics (B. F. Gibson, J. Cohen, R. Chrien, J. Millener, and E. V. Hungerford) .... 23 Physics with a High-Resolution Neutral Meson Spectrometer (J. L. Matthews,

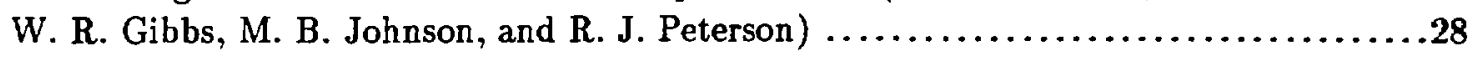

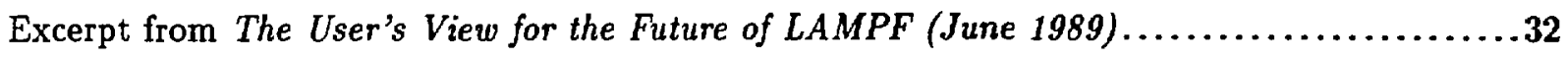
Appendix: Meson Spectroscopy with Light Hadrons (J. R. Comfort and T. Goldman) ........45 


\section{THE USER'S VIEW FOR THE FUTURE OF LAMPF, 1989: REPORTS FROM THE PION PHYSICS WORKING GROUP}

\section{INTRODUCTION}

The Los Alamos Meson Physics Facility has played a dominant role in pion-nucleus physics. This is largely due to its high-intensity beams and the superior resolution of the LAMPF detectors, plus the skill, vitality, and enthusiasm of those who have used them. In the fifteen years or so since these systems began operating, much work has been done, and much has been accomplished, both in experiment and theory. The time has now come to think about where the field now stands and where it should go.

The reports in this document address those questions. They were constructed as part of the long-range planning process for LAMPF which took place in spring, 1989. Motivated by the need for coherent input into the Long Range Plan requested from the Nuclear Science Advisory Committee (NSAC) by the Department of Energy in that year, the LAMPF community formed committees to examine the past accomplishments of LAMPF and to consider its future. Among these was a committee on the physics that is done with pion beams, of which we were co-chairmen. Members of this committee wrote these reports, which served as the basis of a final summary report for NSAC. This final report was produced following meetings of the pion community held at LAMPF, where these reports were read and discussed in some detail.

These input reports reflect the views on the present and the future of pion physics of the many practitioners who helped write them. They are to a great extent the views of the future of pion physics held by the community as a whole. As any final summary report must condense the material to a degree that all detail will be missing, we felt that it is important to preserve the primary reports, which contain the thoughts of many of the experts in the field. They embody the views of the community on where we, as a group, feel that pion-nucleus studies should go. That future, when addressed on its scientific merits, looks bright.

The reports here represent the full range of pion physics that has been studied at LAMPF and other accelerators, and that could be studied at LAMPF in the future, with an appropriate machine upgrade. They begin, appropriately enough, with a discussion of studies of the basic pion-nucleon interaction, as seen in pion-nucleon resonances. Work in this field at LAMPF, for example, has given new insight into the basic characteristics of the first nucleon excited state. To continue these studies to any higher-mass resonances requires higher energies, however, and this report addresses in some detail what might be learned there.

This is followed by discussions of exclusive pion-nucleus reactions and of pion propagation in nuclei as revealed by inclusive reactions. These have perhaps been the principal concern of pion research at LAMPF so far. In this work, new details of nuclear structure have been revealed, mach of it as a result of the simple forms of the pion-nucleon and pion-nucleus interactions in the LAMPF energy region, and much has been learned about the behavior of pions in the nuclear medium. This has not led to a completion of our understanding of these processes, but to a situation in which the 
questions that we are asking are based on previous work and are more detailed rather than more general. There is clearly much to be learned with more studies.

Next, there are considerations of two fields that are either currently inaccessible or barely accessible at LAMPF with its present beam energy. One of these is meson production by pions. This is of great interest, both because of what can be learned about the intrinsic properties of the mesons and of what can be revealed by producing them in nuclei rather than on a single nucleon. The three nonstrange mesons with the lowest masses above the pion are appropriately singled out for discussion, namely the rho, omega, and eta. Studies of only the latter have been carried out at LAMPF, but little more can be done there without beam or accelerator modifications.

The other new field is that of hypernuclear studies, which would be possible at energies around $1 \mathrm{GeV}$ or higher through $(\pi, K)$ reactions. Experiments with these reactions at other laboratories have shown that this is potentially a very fertile method of producing these objects, in which a neutron is replaced by a Lambda (or, perhaps, a nonstrange quark is replaced by a strange quark). Even though hypernuclei have been studied for decades, the surface of what they can tell us about nuclear structure and weak baryon-baryon interactions has only been lightly scratched so far. To continue, what is needed is copious hypernuclear production in a system that permits high-resolution studies, which is possible with a LAMPF upgrade.

The last of these reports discusses the principal new detector system that is currently planned for LAMPF, a new high-resolution neutral meson spectrometer. This device opens the door to several new areas of study. Past limitations on resolution have not allowed us to exploit the variety of states that nature provides to disentangle reaction mechanism from structure. The improved resolution on this spectrometer will allow measurements of reactions which are specifically chosen to provide selective information, often information on the delta-nucleus interaction. It will also allow extended studies of the eta-nucleon and eta-nucleus system.

For the near term, the general feeling of the pion community is that much excellent physics research can be carried out at LAMPF over the next five years or so, using existing or planned facilities. For the long term, the future is clearly at higher energies. Again, the general, but not universal, consensus is that the major requirement is a high-intensity 1-GeV beam, with highresolution detectors, which could be achieved with a 1.6-GeV upgrade of LAMPF. Such a beam would allow studies of hypernuclear physics, meson production and decay, double-charge-exchange scattering at high energies. higher-mass pion-nucleon resonances, and pion-nucleus interactions in the region of these resonances. Such possibilities should mean that the first fifteen years of pion physics at these energies and intensities should be at least as exciting and productive, and with as many surprises, as were the first fifteen years of pion physics at LAMPF.

Another report, included here as an Appendix, is one that was generated as part of the LAMPF long-range planning process, but not directly through the committee structure described above. It considers the meson physics that could be addressed with another attractive option that was discussed, that of a 6-GeV machine. Other physics that could be studied with such a machine is alsc discussed in the report on pion-nucleon interactions. 
The last item in this document (before the Appendix) is an excerpi from the report that was presented to NSAC, which was entitled "The User's View for the Future of LAMPF." This is the section of that report that addresses pion physics, which was based on the reports here.

Finally, we note that the names of the people attached to each report are the primary authors of that report. They deserve many thanks for their efforts, which were completed with deadlines that were much shorter than should exist in a rational world.

George Burleson and David Ernst 


\title{
PION-NUCLEON RESONANCES
}

\author{
M. E. Sadler \\ Abilene Christian University \\ T. Goldman \\ Los Alamos National Laboratory \\ E. R. Kinney \\ Argonne National Laboratory
}

\section{Physics Goals}

QCD provides a precisely defined and well-founded theory of hadronic structure and reactions. Unfortunately, it is all but impossible to reliably calculate its predictions in the nonperturbative regime of low momentum transfers and low-lying spectroscopy, and we must proceed at present by means of models. Nonetheless, QCD does unambiguously predict the existence of exceptional new states: $q^{3}$-plus-gluons and $q^{4}-\bar{q}$ exotic baryons, which cannot be simply described in terms of baryon-meson composites. Testing QCD demands the discovery and detailed examination of such states. However, in order to do so, it is also necessary to obtain a thorough experimental description of nonexotic hadrons. An order-of-magnitude more precise and complete study of $\pi N$ scattering and phase shift analyses is required to understand the nonexotic spectrum with sufficient confidence that the relatively weakly coupled exotica may be fully exposed to view.

Our knowledge of the masses and widths of the nonstrange, isospin-1/2 and $-3 / 2$ baryons comes essentiaily from the partial-wave analysis of $\pi N$ scattering data. It is just these parameters that are predicted by quark, Skyrmion, hybrid or other models of QCD in hadron spectroscopy. Many of the models exhibit remarkable agreement with known low-lying resonances, but many more resonances are predicted than are known to exist. If these models are correct, then either the resonances decouple from the $\pi N$ channel or have not been observed due to lack of experimental data. In any event, inclusive studies of pion-nucleus scattering at the higher energies will certainly need all of the elementary $\pi-N$ cross sections in order to be interpretable.

As the pion energy rises above $1 \mathrm{GeV}$, pion-induced pion production also plays a major role, making up a large fraction of the total $\pi-N$ cross section. It would appear that such inelastic processes are strongly favored by the phase space available in the final state relative to two-body reactions. Understanding the competition of the inelastic processes with exclusive two-body final states will likely be necessary to understand the fundamental reaction dynamics.

The dynamics by which the momentum and energy of the incident pion are shared in a multiple pion final state may be studied much more precisely with intense pion beams in the 1-2-GeV energy range, given the ability to perform studies similar to those planned at CEBAF using electrons. For example, one might be able to use coincidence techniques to study the $\pi-\pi$ interaction by careful restriction of the kinematics and isospin channels. The $\pi-\pi$ interaction is surely one of the fundamental problems to be explained by QCD, beyond questions of hadron structure. Given the $\pi-\pi$ interaction, one can study the distribution of virtual pions around the nucleon, or sea quarks in the language of quark models. The $\pi-\delta$ and $\pi-N^{*}$ interactions might also be probed by careful choice of final state. 
Radiative transitions could be used to study bremsstrahlung of pions scattering in the ficld of the nucleon (or nucleus). These transitions could be interesting because at some point one expects the incoherent radiation from the individual quarks to predominate over the coherent part from the pion.

Another possibility would be to study inclusive pion scattering from the nucleon much the same way as is done with inclusive studies of nuclei. Previous pion-production data is largely from bubble chamber experiments, which typically do not measure the inclusive response over the entire range of outgoing momentum and scattering angle very well. It is also possible that exotic quark-antiquark or gluon states of the nucleon have distinctive inclusive signatures.

\section{Current Status and Needs}

Table I lists the established and weak (marked with * or **) $\pi N$ resonances from the Particle Data Group up to a mass of $3.2 \mathrm{GeV}$, which corresponds to a laboratory pion momentum of $5 \mathrm{GeV} / c$. The primary analyses used for this compilation are those from the Karlsruhe-Helsinkj (K-H) and Carnegie-Mellon-Lawrence-Berkeley (C-L) groups, both of which were last revised in 1980.

The VPI analysis, last published in 1984 but updated regularly via the SAID (Scattering Analysis Interactive Dialin) program, only goes up to $1.2 \mathrm{GeV}$ (pion kinetic energy) and is essentially ignored by the Particle Data Group in determining resonance parameters. Besides the limited energy range, reasons given are that theoretical constraints from dispersion relations and analyticity are not incorporated. Nevertheless, a very interesting feature of the VPI analysis is a double pole for the $P_{11}$ resonance, at positions $(1359,-100 i)$ and $(1410,-80 i)$ in the complex plane (in MeV). A double $P_{11}$ was also reported in the Saclay 1974 analysis.

Cutkosky, one of the collaborators on the C-L analysis, offered the following possibilities on the (possible) structure of the $P_{11}$ in the Second International Symposium on $\pi N$ Physics held in Los Alamos in 1987:

1. "...that some new degree of freedom is being activated, and that this, by accident, happens to occur at the same energy where one of the quark-model states is found."

2. "...that the forces among the quarks might lead to a significant amount of diquark clustering in baryons."

3. "...that the narrow-resonance approximation is very poor for the $P_{11}(1440)$, and the coupling to various open channels has an important influence on its properties. If this view is correct, we shall have to admit that $q^{4} \bar{q}$ and $q^{5} \bar{q}^{2}$ configurations are important, as well as $q^{3}$ configurations."

However, he concluded that "the most likely interpretation of the AFR (Arndt-Ford-Roper) two-pole fit to the $P_{11}$ resonance is rather banal-the two poles are just what one expects." A single resonance can have more than one pole in the presence of open inelastic channels. If this conclusion is correct some explanation is also in order as to why the nearby $S_{11}$ and $D_{13}$ resonances do not exhibit this "shadow" pole. 
The UCLA-ACU-GWU collaboration has obtained complete data sets $\left(d \sigma / d \Omega, P=A_{N}\right.$, and spin rolation parameters $A$ and $R$ for $\pi^{+} p \rightarrow \pi^{+} p$ and $\pi^{-} p \rightarrow \pi^{-} p$; and $d \sigma / d \Omega$ and $A_{N}$ for $\left.\pi^{\rightarrow} p \rightarrow \pi^{0} n\right)$ up to the $P_{11}$, which is the present limit of the pion beam energy at LAMPF. These data, when incorporated into the analyses, should help settle the controversy, but it is obvious that complete data sets above the $P_{11}$ are also desired.

Such a modest increase could be accomplished by a PILAC in the existing $\mathrm{P}^{3}$ channel. Indeed, a PILAC that would boost the attainable energy to $1-1.3 \mathrm{GeV}$ would cover the low-lying $D_{13}$ and $S_{11}$ resonances and the cluster of resonances in the mass range of 1.6-1.7 GeV. Here, six established (3or 4-star) isospin-1/2 resonances are essentially degenerate when the uncertainties in their masses and widths are taken into account. Better $\pi^{-} p$ data (both elastic scattering and charge exchange) are sorely needed in this energy range in order to disentangle these states. The situation is further clouded because questionable charge-exchange data from the Rutherford Laboratory were heavily used by both the K-H and C-L efforts. These data apparently need to be shifted by $\sim 5 \%$ in nomentum, and, moreover, the backward-angle analyzing-power data lead to violations of isospin variance by three standard deviations when combined with the recent accurate elastic data from the Leningrad and LAMPF groups. The LAMPF charge-exchange data are consistent with isospin invariance and do not agree with the Rutherford data at the back angles near $P_{\pi}=650-700 \mathrm{MeV} / \mathrm{c}$. This momentum is just where things are starting to get interesting.

Another cluster of six established resonances occurs near a mass of $1.9 \mathrm{GeV}$, but this time it is in the isospin-3/2 channel. Again, these states are essentially degenerate in their complex pole positions, considering the uncertainties in their masses and widths. If the one-star candidate for the $D_{33}$ is included, then all quantum numbers through the $F$ wave are exhausted. Is this an accident or is nature trying to tell us something? (It should be pointed out here that David Bugg recently presented similar papers at the Eighth International Symposium on High Energy Spin Physics [Minnesota] and the Third International Symposium on $\pi N$ Physics [Leningrad] in which he attributed the apparent clustering of resonances to their being "triggered" by the rapid opening of inelastic channels.) This energy region is outside that which can be attained with the proposed PILAC and pushes the limits of what can be attained with a 1.6-GeV proton beam using an afterburner linac. A 6-GeV circular machine would allow a study of all resonances listed in Table $I$. A 100-mA primary proton beam would make a systematic study of resonance parameters possible in a reasnnable amount of time. The amount of beam time would be considerable to cover this energy range at appropriate intervals with complete angular distributions for all measurements ( $d \sigma / d \Omega$, $A_{N}, A$, and $R$ ) for all three reactions. Measurements should be made at intervals of $0.02-0.05 \mathrm{GeV}$ in $\sqrt{s}$ (20-50 energies per $\mathrm{GeV}$ interval), with the spacing increasing at increasing energy due to the resonances becoming broader. However, complete sets of measurements are probably needed at only a few energies in order to test the theoretical constraints applied in partial-wave analyses, to resolve continuum ambiguities, and to provide redundancy in the data sets. Appropriate interaction between experimentalists, theorists, and partial-wave analysts will be needed to optimize the ratio of (physics obtained)/(a * dollars spent $+b *$ sweat produced $)$. The physics to be obtained is substantial. One is hard pressed to pose more fundamental questions than "what holds the quarks together?," "where are the gluon excitations?," or "where is the exotic matter, i.e, $q^{4} \bar{q}$, etc.?" 
Table I. List of $N^{*}$ and $\Delta$ iesonances with the corresponding laboratory pion moment um (column 1). kinetic energy (column 2), and center-of-mass energy (column 3) for creation via $\pi p$ scattering. Columns 4 and 5 list the $N^{*}$ and $\Delta$ resonances from the Particle Data Group at the appropriate energy, with * indicating "evidence weak, likely to disappear" and ** indicating "not established, needs confirmation." The resonances are listed in the traditional $L_{2 I, 2 J}$ format.

\begin{tabular}{|c|c|c|c|c|}
\hline $\begin{array}{c}P_{\mathrm{lab}} \\
(\mathrm{GeV} / c)\end{array}$ & $\begin{array}{c}T_{\mathrm{lab}} \\
(\mathrm{GeV})\end{array}$ & $\begin{array}{c}\sqrt{s} \\
(\mathrm{GeV})\end{array}$ & $N^{*}(I=1 / 2)$ & $\Delta(I=3 / 2)$ \\
\hline 0.50 & 0.38 & 1.37 & & \\
\hline 0.60 & 0.48 & 1.43 & $P_{11}$ & \\
\hline 0.70 & 0.57 & 1.50 & $D_{13}, S_{11}, P_{13} *$ & $P_{31}^{*}$ \\
\hline 0.80 & 0.67 & 1.56 & & $P_{33}^{* *}$ \\
\hline 0.90 & 0.77 & 1.62 & $S_{11}$ & $S_{31}$ \\
\hline 1.00 & 0.87 & 1.67 & $D_{15}, F_{15}, D_{13}, P_{11}, P_{13}$ & $D_{33}$ \\
\hline 1.10 & 0.97 & 1.73 & & \\
\hline 1.20 & 1.07 & 1.78 & & \\
\hline 1.30 & 1.17 & 1.83 & & \\
\hline 1.40 & 1.27 & 1.88 & & $S_{31}, F_{35}, P_{31}, P_{33}$ \\
\hline 1.50 & 1.37 & 1.93 & $?(1960)^{*}$ & $D_{35}, D_{33}{ }^{*}, F_{37}$ \\
\hline 1.60 & 1.47 & 1.98 & $F_{17}^{* *}, F_{15}^{* *}$ & $F_{35} * *$ \\
\hline 1.70 & 1.57 & 2.03 & & \\
\hline 1.80 & 1.67 & 2.07 & $D_{13}{ }^{* *}, S_{11}{ }^{*}, P_{11}{ }^{*}$ & \\
\hline 1.90 & 1.77 & 2.12 & & $S_{31}^{*}$ \\
\hline 2.00 & 1.87 & 2.16 & $G_{17}$ & \\
\hline 2.10 & 1.97 & 2.20 & $D_{15}{ }^{* *}, H_{19}$ & $G_{37}^{*}$ \\
\hline 2.20 & 2.06 & 2.24 & $G_{19}$ & \\
\hline 2.30 & 2.16 & 2.29 & & $H_{39}{ }^{* *}$ \\
\hline 2.40 & 2.26 & 2.33 & & $D_{35}^{*}$ \\
\hline 2.50 & 2.36 & 2.37 & & $F_{37^{*}}, G_{39}{ }^{* *}$ \\
\hline 2.60 & 2.46 & 2.41 & & $H_{311}$ \\
\hline 2.70 & 2.56 & 2.44 & & \\
\hline 2.80 & 2.66 & 2.48 & & \\
\hline 2.90 & 2.76 & 2.52 & & \\
\hline 3.00 & 2.86 & 2.56 & & \\
\hline 3.10 & 2.96 & 2.59 & $I_{111}$ & \\
\hline 3.20 & 3.06 & 2.63 & & \\
\hline 3.30 & 3.16 & 2.66 & & \\
\hline 3.40 & 3.26 & 2.70 & $K_{113}{ }^{* *}$ & \\
\hline 3.50 & 3.36 & 2.73 & & $I_{313} * *$ \\
\hline 3.60 & 3.46 & 2.77 & & \\
\hline 3.70 & 3.56 & 2.80 & & \\
\hline 3.80 & 3.66 & 2.83 & & \\
\hline 3.90 & 3.76 & 2.87 & & \\
\hline 4.00 & 3.86 & 2.90 & & \\
\hline 4.10 & 3.96 & 2.93 & & $\kappa_{315} * *$ \\
\hline 4.20 & 4.06 & 2.96 & & \\
\hline
\end{tabular}


Table I. (continued)

\begin{tabular}{ccccc}
\hline $\begin{array}{c}P_{\text {lab }} \\
(\mathrm{GeV} / c)\end{array}$ & $\begin{array}{c}T_{\text {lab }} \\
(\mathrm{GeV})\end{array}$ & $\begin{array}{c}\sqrt{s} \\
(\mathrm{GeV})\end{array}$ & $N^{*}(I=1 / 2)$ & $\Delta(I=3 / 2)$ \\
\hline 4.30 & 4.16 & 3.00 & & \\
4.40 & 4.26 & 3.03 & \\
4.50 & 4.36 & 3.06 & \\
4.60 & 4.46 & 3.09 & \\
4.70 & 4.56 & 3.12 & \\
4.80 & 4.66 & 3.15 & \\
4.90 & 4.76 & 3.18 & \\
5.00 & 4.86 & 3.21 & \\
\hline \hline
\end{tabular}

\section{Experimental Considerations}

Before embarking on a systematic program as described in the previous section, an appraisal is needed of what remains to be measured or needs to be remeasured. In general, the $\pi^{-} p$ data (both elastic and charge exchange) are in worse shape than the $\pi^{+} p$ data. No data for the spin rotation parameters exist between 0.7 and $6 \mathrm{GeV} / c$ (laboratory pion momentum). It needs to be emphasized that careful evaluation of systematic uncertainties is essential to any of the measurements in the program. These data should become the standard for decades (centuries?) to come not only for their inherent importance but also for normalizing other data sets (e.g., pion scattering on nuclear targets).

Complete angular distributions of differential cross sections or analyzing powers are needed. The highest power of $\cos \theta$ (or Legendre polynomial) required to parameterize the angular distribution of these observables goes as $2 \times L$, where $L$ is the highest-order partial wave contributing to the reaction. Even for the low-lying resonances (which go up to $F$ waves) a minimum of 15 angular measurements, evenly distributed over $-1<\cos \theta<1$, are needed in order to evaluate the contributions from higher partial waves.

Measurements at the extremes of the angular distributions (near $0^{\circ}$ and $180^{\circ}$ ) are needed. Higher-order partial waves contribute most significantly in this region. Measurements in the coulomb interference region allow determination of the relative phase of the coulomb and hadron amplitudes. However, isolated measurements over a limited angular range are of limited use in a. partial-wave analysis. Any measurement should extend far enough in energy or angle to overlap with other measurements in order to evaluate the systematic errors (i.e., momentum shifts and normalizations).

A complete re-evaluation of cross sections and analyzing powers for $\pi^{-} p \rightarrow \pi^{0} n$ appears to be in order due to the paucity of data between $0.7 \mathrm{GeV} / c$ and $1 \mathrm{GeV} / c$ and above $2 \mathrm{GeV} / c$, discrepancies between data sets throughout the resonance region, and difficulties in fitting the present data with existing partial-wave analyses, which rely upon isospin invariance of the amplitudes. The proposed neutral meson spectrometer can contribute significantly in this effort, as described in one of the next reports. 
Total cross sections for both $\pi^{+} p$ and $\pi^{-} p$ need to be remeasured throughout the resonance region. Existing data ${ }^{\mathbf{1}, 2}$ are in disagreement, some but not all of which can be resolved by a simple relative shift of the respective momenta. A high-duty-factor machine is highly desired for these measurements, which entail counting the beam. Indeed, once the apparatus is available, the measurements should extend down to $0.4 \mathrm{GeV} / \mathrm{c}$ to complement the LAMPF program just completed. Total cross sections were not miade in this program due to the poor duty factor and the limited momentum range available.

Data on $\pi N \rightarrow$ other states are needed in evaluating inelasticities of resonances and because the opening of inelastic channels can produce resonance-like behavior (cusps in Argand diagrams) in the partial waves. There is appreciable cross section to inelastic channels even at $0.6 \mathrm{GeV} / c$. Capability to measure multiple-pion final states will be needed. Programs which should proceed in step with the one described here are $\pi N \rightarrow$ other two-body states and quasi-two-body states $(\eta N$, $\rho N, K \Lambda, K \Sigma, \omega N, \phi N, \rho \Delta, \ldots)$. Indeed, with a hadron factory with intense proton beams of energy to $6 \mathrm{GeV}$, enough data on these channels could be obtained to warrant their own phase shift analyses. Besides contributing to the elastic analyses, such analyses would provide information on resonances which couple weakly to $\pi N$ elastic scattering.

Besides higher energy pions ( $>1 \mathrm{GeV}$ ), one will need new detection systems to carry out the suggested inelastic measurements. Whether the measurements are made with a combination of magnetic spectrometers, a large acceptance detector, or some combination of magnetic spectrometer and other detectors, must be carefully studied, with the goals of particular measurements in mind. CEBAF will provide the capability to investigate the resonance region using photo- and electroproduction. Pion-nucleon scattering should provide a great deal of complementary information to that obtained at CEBAF as well as move us far beyond the bubble chamber studies of a decade ago.

Momentum is gathering for an assault on the determination of the resonance properties, and an intense hadron facility is needed on the front line.

\section{References}

1. A. A. Carter et al., Phys. Rev. 168, 1457 (1968).

2. V. Davidson et al., Phys. Rev. D5, 499 (1972). 


\title{
EXCLUSIVE REACTIONS
}

\author{
D. D. Strottmain \\ Los Alamos National Laboratory \\ H. T. Fortune \\ University of Pennsylvania
}

In the last half decade the pion has come of age and is now an established tool in the quest to explore and understand the nucleus. In addition to its essential role in elucidating the behavior of the delta in nuclear matter, it has revealed nuclear modes of excitation previously unknown. Its properties as a probe of nuclear structure are complementary to those of the electron and the nucleon, in that each is sensitive to different aspects of nuclear structure.

\section{Accomplishments Over the Past Five Years}

Among the unique properties of the pion as a probe is the isospin; it occurs in three charge states and is the only elementary probe that allows a study of double charge exchange. This permits an investigation of levels with exotic quantum numbers or states that vould otherwise be buried in the background. One such example is the double dipole excitation. Although examples of giant resonances built upon the first excited level have been seen in $(p, \gamma)$ reactions, ${ }^{1}$ there had been no resonances observed that were built upon highly excited levels. Using pion-induced double charge exchange (DCX), one has now observed ${ }^{2}$ giant dipole resonances (GDR) built upon the isobaric analog state and also upon the giant dipole resonance itself (GDR2). This latter state may have an angular momentum of either zero or two, although to date only the latter has been observed.

At low bombarding energies the surprising result was found ${ }^{3}$ that the observed cross sections in DCX are appreciably larger than anticipated from results of single charge exchange (SCX). Although this led to speculation regarding possible observation of six-quark bags, ${ }^{4}$ other mechanisms including double spin flip could also account for the observed cross section. In particular, it has been shown that the effects seen can be produced by the spatial correlations in the nuclear wave functions. ${ }^{5,6}$ Theoretical analyses have provided a decomposition of the double scattering amplitude into short- and lorg-range components, which explain both analog and ground-state transitions.

Pion DCX has also been used to investigate the role of the $\Delta(1232)$ in pion-induced reactions and to search for exotic particles. Many mechanisms ${ }^{7}$ may contribute to $\left(\pi^{+}, \pi^{-}\right)$; it has been established that there are at least two essential mechanisms in the resonance region ${ }^{8}$ and that they are out of phase by $180^{\circ}$ at $164 \mathrm{MeV}$ and by $90^{\circ}$ at $300 \mathrm{MeV}$. DCX experiments have also set an extremely low limit ${ }^{9}$ of about $0.3 \mathrm{nb} / \mathrm{sr} / \mathrm{MeV}$ for detection of an isospin-two dibaryon object. It may also provide a connection with double beta decay since both processes require information on nucleon-nucleon correlations. ${ }^{10}$

The use of pion-induced single charge exchange led to the discovery and investigation of the isovector monopole state. ${ }^{11}$ The SCX reaction on aligned ${ }^{165}$ Ho has been used to determine the deformation of the neutron distribution. ${ }^{12,13}$ Measurements of precise elastic scattering cross sections between far-forward and far-backward angles have led to a better understanding of second-order effects in the optical potential description of pion-nucleus interactions. 
Pion inelastic scattering has proved to be a pov/erful tool for nuclear spectroscopy. The nineto-one ratio of $\pi^{+}$to $\pi^{-}$cross sections for scattering from protons (and vice-versa for neutrons) provides a method to ascertain the proton and ne'stron admixture in nuclear wave functions. The neutron and proton matrix elements so determined have been found to agree with those obtained from electromagnetic transitions in the cases where the transitions are known in isobaric nuclei. ${ }^{14}$ Thus, one has a preeminent tool, which still needs to be fully exploited, to obtain both proton and neutron matrix elements in neutron-rich nuclei.

Measurements ${ }^{15}$ of analog transitions in SCX have produced data for energies between 20 and $500 \mathrm{MeV}$ on nuclei from ${ }^{3} \mathrm{He}$ to ${ }^{208} \mathrm{~Pb}$. These data constitute the empirical basis for the theoretical descriptions of the isovector component of the pion-nucleus optical potential. At pion energies near $50 \mathrm{MeV}$ these cross sections were found to exhibit a very sharp minimum, a feature that is evidence for high nuclear transparency and for the validity of the impulse approximation.

\section{Physics Over the Next Ten Years}

For the majority of experimental studies, further development will require both higher flux and higher energies. The principal exception to the need for higher intensities is for experiments involving polarized targets, which cannot generally function long in a high flux environment. Experiments studying elastic, charge-exchange, and inelastic scattering on polarized nuclear targets are just beginning. They should produce important information on the spin dependence of the Deltanucleus interaction, on the spin structure of excited states, and on pion-nucleus spin-dependent amplitudes in general. The DCX reaction in particular is now severely limited by the count rate. Higher intensities will allow better statistics for weakly-seen states, as well as the possibility to seek additional modes of excitation for which running time at the current facilities is prohibitive. Observation of excited levels in DCX will greatly help determine the reaction mechanisms at both low and resonance energies. The use of the $\left(\pi^{-}, \pi^{+}\right)$reaction has been demonstrated to be an excellent method to measure the masses of neutron-rich nuclei, ${ }^{16}$ but its effective application has been severely limited by statistics. The need for higher intensities is most apparent above the $\triangle(1232)$ resonance. Although LAMPF is the only pion factory currently able to reach these energies, experiments above $550 \mathrm{MeV}$ become impractical. Higher intensities would also allow the use of either thinner targets, when required by a need for higher energy resolution, or of targets of exotic material for which there are smaller amounts of target material available.

The energy region above the $\Delta(1232)$ resonance shows great promise. The GDR2 state has only been seen for $290-\mathrm{MeV}$ pions, ${ }^{17}$ whereas preliminary searches at 164 and $230 \mathrm{MeV}$ were unsuccessful. Since the peak of the background continuum moves away from the region of interest as the beam energy increases, searching for such states could be quite fruitful at higher energies if there is sufficient intensity. In addition to the unobserved $0^{+}$member of the GDR2, other exotic modes of excitation should exist and should be sought. Among these are the giant monopole resonance based upon the giant monopole (GMR2) and the giant quadrupole based on the isobaric analog state (GDR4-IAS). Observation of these states would shed considerable light on large-amplitude excitations of the nucleus.

As one moves into higher energies, one enters a new era. Below $1.5 \mathrm{GeV}$ there are energy regions in which the pion-nucleon phase shifts are poorly known; between 1.5 and $6 \mathrm{GeV}$ even 
less is known. The famous nine-to-one $\pi^{+}$to $\pi^{-}$ratio around the $\Delta(1232)$ resonance reverses at higher energy. The interesting $\left(\pi^{ \pm}, \eta\right)$ reaction also becomes possible, as does DCX, through an intermediate $\eta$ or $\rho$. Each of these acts as a filter and will probe different nucleon degrees of freedom. They will be developed into valuable tools if appropriate detection schemes are available. If the eta has a bound state, an entirely new field of spectroscopy opens. The $\left(\pi^{ \pm}, \eta\right)$ SCX reaction proceeds via $\Delta T=1$, so the reaction is more selective than is $\left(\pi^{ \pm}, \pi^{0}\right)$. This reaction will also provide another probe to explore the high-momentum components of the wave functions. The $\left(\pi^{ \pm}, \rho^{0}\right)$ reaction will select the $\Delta S=1, \Delta T=0,1,2$ piece of the charge exchange process and will complement the $\left(\pi^{ \pm}, \pi^{0}\right)$ reaction.

Above the $\Delta(1232)$ resonance, both SCX and DCX cross sections begin to increase. Above $600 \mathrm{MeV}$ the forward cross sections are predicted to be roughly constant until around $900 \mathrm{MeV}$. Between $900 \mathrm{MeV}$ and $1.2 \mathrm{GeV}$, the $\mathrm{DCX}$ cross section is predicted ${ }^{18}$ to drop by four orders of magnitude if the process is assumed to proceed via a sequential mechanism. Thus, around $1 \mathrm{GeV}$ should be an excellent place to search for new reaction mechanisms or short-range correlations such as six-quark bags.

At pion energies between 0.7 and $1.5 \mathrm{GeV}$, eta meson production by pions becomes abundant; the total cross section for $\pi p \rightarrow \eta n$ reaches several millibarns. Because the eta has isospin zero and the eta-nucleon channel is strongly coupled to the $S_{11} \pi N$ resonance $N^{*}(1535)$, etas are ideal to study the $N^{*}$. Unlike the $\Delta(1232)$, the $N^{*}(1535)$ has one of its quarks in an excited orbital ${ }^{19}$ or is a four quark-one antiquark state. ${ }^{20}$

Using the experimental decay branching ratios of $N^{*}(1535)$ to $\pi N$ and $\eta N$ channels, one can show that the $\eta N N^{*}$ coupling constant is a factor of two greater than the $\pi N^{*}$ coupling constant, in marked contrast to the fact that the $\eta N N$ coupling constant is a factor of three smaller than the $\pi N N$ coupling constant. This reversed ratio in the coupling constants indicates that the meson dynamics involving $N^{*}(1535)$ are very different from those involving the nucleon. Although the contributions of the eta meson to nuclear structure and low-energy $N$ - $N$ scattering can be neglected to a good approximation, the eta dynamics must be taken into account in the study of the $N$-N interaction at energies above the eta production threshold.

\section{Facilities Needed Over the Next Five Years}

Over the past decade the pion physics community has been served exceedingly well by the high-intensity, high-resolution pion beams available at LAMPF, which are unique. The EPICS spectrometer system has the best resolution in the world (about $150 \mathrm{keV}$ ). The recent modification of the $\mathrm{P}^{3}$ channel has made it possible for good-resolution studies to be carried out at energies up to $550 \mathrm{MeV}$. The Clamshell spectrometer in the Low Energy Pion Channel will be exploited more fully after the energy compressor ("scruncher") system has been installed, which should increase counting rates by about a factor of five. The resolution of the existing pi-zero spectrometer has for sometime been felt to be clearly inadequate, though some of the exciting results at LAMPF were accomplished with this device. Speedy construction of a good resolution (200-250 keV) neutral meson spectrometer (NMS) is thus of the highest priority. This device would allow SCX measurements to be extended to nonanalog states, including spin-flip transitions involving GamowTeller resonance. In addition, it is absolutely essential to keep the existing pion facilities at LAMPF 
operating full time. Many of the accomplishments of the past five years have only scratched the surface of a rich field of new nuclear phenomena which were not accessible prior to the construction of the meson factories. In addition, questions that will be raised by a new NMS will demand complementary experiments with charged pions.

\section{Facilities Needed Over the Next Ten Years}

The next step in a further exploitation of the pion in the study of nuclear (and nucleon) structure requires pion beams of about $10^{9} / \mathrm{s}$ into $\Delta p / p \sim 1 \%$ between $\Delta(1232)$ resonance energies and about $1 \mathrm{GeV}$. This can be accomplished as an upgrade of LAMPF by increasing the proton energy and the primary proton flux to about $2 \mathrm{~mA}$. An improvement in duty factor would greatly facilitate coincidence experiments. New detectors with high efficiency and good energy resolution and coincidence capabilities would be needed. The proposed new NMS would be an important detector at such an upgraded facility. An appropriate detector for charged particies would also be needed (EPICS II). The parameters of this device should provide an energy resolution similar to that of EPICS (but at $1 \mathrm{GeV}$ ). The investigation of the new excitation modes of the nucleus using inelastic scattering and DCX will require both high efficiency (or a large momentum bite) and an energy resolution of $100-200 \mathrm{keV}$.

\section{References}

1. K. Snover, Ann. Rev. Nucl. Part. Phys. 36, 545 (1987).

2. S. Mordechai et al., Phys. Rev. Lett. 60, 531 (1988); ibid, 61, 531 (1988).

3. M. Leitch, "Low-Energy Pion Double Charge Exchange and Nucleon-Nucleon Correlations in Nuclei," Workshop on Nuclear Structure with Medium-Energy Probes, Santa Fe, New Mexico, October 12, 1988, Los Alamos National Laboratory document LA-UR-88-3854; Bull. Am. Phys. Soc. 33, 1548 (1988).

4. G. A. Miller, Phys. Rev. Lett. 53, 2008 (1984).

5. N. Auerbach et al., Phys. Rev. Lett. 59, 1076 (1987); N. Auerbach et al., Phys. Rev. C38, 1277 (1988).

6. M. Bleszynski and R. J. Glauber, Phys. Rev. C36, 681 (1987).

7. E. Oset, talk presented at Symposium on Delta-Nucleus Dynamics, Argonne National Laboratory (1983).

8. H. T. Fortune and R. Gilman, Phys. Rev. C33, 2171 (1986).

9. D. Ashery et al., Phys. Lett. B215, 41 (1988), and to be published.

10. A. Fazely and L. C. Liu, Phys. Rev. Lett. 57, 968 (1986).

11. J. D. Bowman, Can. J. Phys. 65, 654 (1987).

12. J. N. Knudson et al., Phys. Rev. C35, 1382 (1988).

13. J. Bartel et al., to be published.

14. For a summary of the status and references, cf. D. Strottman, Ref. 3, p. 1549.

15. S. Rokni et al., Phys. Lett. B202, 35 (1988).

16. K. K. Seth et al., Phys. Lett. B173, 397 (1986).

17. H. T. Fortune, private communication.

18. E. Oset and D. St,ottman, to be published.

19. T. DeGrand and R. L. Jaffe, Ann. Phys. (NY) 100, 425 (1976).

20. D. Strottman, Phys. Rev. D 20, 748 (1979). 


\title{
PION PROPAGATION IN NUCLEI
}

\author{
J. L. Mat thews and R. P. Redwine \\ Massachusetts Institute of Technology \\ W. R. Gibbs \\ Los Alamos National Laboratory
}

\section{Introduction}

Considerable progress has been made over the last decade at the three meson facilities (LAMPF, TRIUMF, PSI) in understanding how a pion propagates in the nuclear medium; however, many details are not yet known. The pion is a strongly interacting particle; like the nucleon, it has a significant probability for interacting more than once within a nucleus. In contrast to the nucleon, however, the pion is a boson, so that its absorption and production can be studied. Pion absorption is an important part of any pion propagation, and pion production is an inseparable part of any nucleon-nucleon interaction.

\section{Pion Absorption}

Both experimental and theoretical work on the total and differential cross sections and spin observables for the fundamental pion absorption reaction $\pi+d \rightarrow N+N$ and its inverse has continued during the past five years. This work has yielded substantial improvements in both the characterization of the measurea observables and the microscopic description of the phenomena associated with the reaction for incident pion energies below $300 \mathrm{MeV}$.

Although the pion can be absorbed by a single (off-shell) nucleon in a nucleus, energy and momentum conservation strongly favors absorption by at least two nucleons. The existence of a two-nucleon absorption mechanism in nuclei has been clearly established in both inclusive and exclusive measurements. ${ }^{1-4}$ In addition, studies ${ }^{5-8}$ of the ${ }^{3} \mathrm{He}\left(\pi^{+}, p p\right) p$ and ${ }^{3} \mathrm{He}\left(\pi^{-}, p n\right) n$ reactions conclusively demonstrate the suppression of absorption on $T=1$ nucleon pairs by at least an order of magnitude. This is now understood in terms of the dominance of the $\Delta$ resonance. However, there is evidence ${ }^{2,3}$ that a significant part of the pion absorption cross section cannot be explained by two-nucleon absorption, even when initial- and final-state interactions are included. Signatures of the participation of three nucleons in pion absorption on light nuclei near the $\Delta$ resonance have now been observed unambiguously by several groups. ${ }^{9-11}$ Evidence for processes involving more than three nucleons is unclear. Experiments are both underway and planned at PSI, ${ }^{i 2}$ LAMPF, ${ }^{13}$ and TRIUMF $^{14}$ to obtain more information on a possible coherent multi-nucleon absorption mechanism.

Assuming that the absorption process does hold surprises for us, it will be very important to use higher-energy pions for such studies. By going to higher energies one can increase the "mean free path" of the pion in the nucleus, thus studying the interaction of the pion over a larger fraction of the nuclear volume. The results of these experiments will be complementary to those of photon absorption experiments which are planned at CEBAF and other electromagnetic facilities. Higher pion energies will also allow exploration of the absorption process through the higher-energy baryon resonance region. 


\section{Double Charge Exchange}

Another process which must involve at least two nucleons is piol $_{2}$ double charge exchange: $\left(\pi^{ \pm}, \pi^{\mp}\right)(\mathrm{DCX})$. A large amount of high-quality, systematic data on inclusive DCX has been obtained at LAMPF for nuclei ranging from ${ }^{3} \mathrm{He}$ to ${ }^{208} \mathrm{~Pb}$, at incident pion energies in the $\Delta$ resonance region..$^{15-20}$ In the lightest nuclei, the energy distribution of the outgoing pions showed an unexpected double-peaked structure. ${ }^{18}$ In heavy nuclei, the cross section for the $\left(\pi^{-}, \pi^{+}\right)$reaction appears to "saturate," in contrast to that for the $\left(\pi^{+}, \pi^{-}\right)$reaction which increases monotonically with mass number. ${ }^{21}$ Both of these observations turn out to be consistent with a model in which sequential single charge exchange (SCX) proceeds in competition with other, more probable reactions such as quasifree scattering and absorption..$^{20,21}$

At higher energies, other DCX mechanisms such as scattering from an exchange pion, ${ }^{22}$ or pion-induced pion production followed by absorption of the "extra" pion, ${ }^{23}$ are expected to become important, but the dominant mechanisms are not yet known. One of the interesting open questions is the importance of triple scattering processes, ${ }^{15}$ and how this contribution varies with energy and mass number. At energies above the $\Delta$ there are essentially no data on the inclusive DCX reaction.

\section{Quasifree Nucleon Knockout}

One of the real successes of pion-nucleus physics has been the use of quasifree reactions to study the microscopic interactions of pions and isobars in the nucleus. The best example is the isolation of the contribution to the coincidence $(\pi, \pi N)$ cross section of the process in which a $\Delta$ is formed and then knocks out a nucleon. ${ }^{24}$ An important part of these studies was done at LAMPF using the $\pi^{0}$ spectrometer and an array of plastic scintillators to observe the ${ }^{16} \mathrm{O}\left(\pi^{+}, \pi^{0} p\right)$ reaction. ${ }^{25}$

A more complex process is that in which the pion knocks out two nucleons $[(\pi, \pi N N)]$. An experimental investigation of this reaction will provide a means of studying several important and unsettled issues in nuclear physics: (1) The propagation of the fully off-shell intermediate pion can be examined over an easily tunable range of invariant mass and momentum transfer. (2) Shortrange spatial correlations of nucleons in the target nucleus can be inferred from the correlations in the final state of nucleons at large relative momentum. (3) By selecting various combinations of $T_{3}(\pi)=0, \pm 1$ and $T_{3}(N)= \pm 1 / 2$ and a $T=0$ target, one can observe the interference of all three isospin amplitudes that are accessible to the $\pi N N$ system. The first experimental study of the $(\pi, \pi N N)$ reaction has been approved to run at LAMPF. ${ }^{26}$

The $\left(e, e^{\prime} N N\right)$ processes have long been viewed as an important component of the program at high-energy, high-duty-factor electron accelerators such as CEBAF. While in principle the results of the electrc nagnetic measurements will be more readily interpretable in terms of $N \boldsymbol{N}$ correlations, the $(\pi, \pi N N)$ reaction, with its larger cross section, will be experimentally easier. As in many other cases, the pion- and electron-induced reactions will provide valuable complementary information.

It will be important to study the quasifree processes at higher pion energies. The greater penetrability of the pions in the nuclear medium will be an advantage, as will be the better kinematic separation between various mechanisms. In addition, one will have the opportunity to investigate the interactions of the higher-energy baryon resonances with the nuclear environment. 


\section{Meson Production}

One of the most interesting issues in modern nuclear physics revolves around the distinction between classical and coherent quantum propagation of hadrons through the nuclear medium. The study of pion propagation in the energy region where $(\pi, 2 \pi),(\pi, \eta),(\pi, \rho)$, etc., reactions are possible provides an opportunity to investigate this process in a controlled environment. ${ }^{27}$ The role of the production of these mesons ${ }^{28}$ (virtual or real) is an important issue. At present, there is only very meager experimental information on the pion-induced pion production $[(\pi, 2 \pi),(\pi, 3 \pi)$, ...] reactions. Recent experiments on ${ }^{16} \mathrm{O}$ and ${ }^{12} \mathrm{C}$ at $\mathrm{TRIUMF}^{29}$ and $\mathrm{PSI}^{30}$ yield contradictory results.

A specific example of a two-step reaction involving the $\eta$ which could be investigated is $\eta$ production followed by pion production by the previously produced $\eta$ (see Ref. 27). The final resulting pion can be distinguished from other pions (if the $\eta$ is real) by kinematics. Thus by observing this reaction near its threshold, one could follow the process and study its mechanism from on- to off-shell conditions.

The production of vector mesons has been suggested as a diagnostic tool for relativistic heavyion reactions. At the onset of a phase transition from hadronic matter to a quark-gluon plasma, the ratio of the cross section for the threshold production of $\phi$ mesons to that for $\rho$ 's or $\omega$ 's is predicted to increase abruptly. ${ }^{31}$ Clearly, an understanding of the production rate and the propagation properties of these mesons around threshold is essential for the interpretation of these experiments.

Since the $\rho, \omega$, and $\phi$ mesons decay strongly, one must infer their presence by detecting two or three pions (or, in the case of the $\phi$, kaons) in the final state.

\section{New Instrumentation}

Many of the reactions discussed above require, and most would be further elucidated by, detection of several particles in the final state. Ideally, one would want a detector with an acceptance of $4 \pi$ steradians which could energy-analyze mesons (of at least both positive and negative, if not zero, charge), protons, and neutrons. Detectors which satisfy some of these requirements are currently in use or being constructed at LAMPF (the BGO Ball-an essentially $4 \pi$ array of bismuth germanate crystals), ${ }^{32}$ PSI (LADS-a Large Acceptance Detector System of wire chambers and plastic scintillators), ${ }^{12}$ and TRIUMF (CHAOS-Circular High Acceptance Orbit [magnetic] Spectrometer) ${ }^{33}$ The accelerators at PSI and TRIUMF have the advantage over LAMPF of higher duty factor; however, LAMPF has the advantage already of higher energy, and is actively considering an energy increase. Even without increased duty, the higher-energy pion beams, with a detector utilizing a "clever trigger" and new fast-event processing techniques, will make LAMPF fully competitive in this field. An increase in the duty factor would, needless to say, be highly desirable.

\section{Summary}

Given the isospin options available with pion beams, one can explore the full isospin content of the nuclear response by means of quasifree scattering, SCX, and DCX measurements. This program becomes yet more interesting the higher the beam energy one uses, and matches closely the goals 
of recent and proposed electromagnetic and nucleon-induced reactions. The quantum numbers of the pion $(S=0, T=1)$ give a different insight into this very general program. In addition, the baryon number $(B=0)$ of the pion allows the processes of absorption and production to occur, whose understanding is essential for the understanding of the nucleon-nucleon interaction in nuciei.

\section{References}

1. R. D. McKeown et al., Phys. Rev. C24, 211 (1981).

2. A. Altman et al., Phys. Rev. Lett. 50, 1187 (1983); Phys. Rev. C34, 1757 (1986).

3. W. J. Burger et al., Phys. Rev. Lett. 57, 58 (1986).

4. R. A. Schumacher et al., Phys. Rev. C38, 2205 (1988).

5. D. Ashery et al., Phys. Rev. Lett. 47, 895 (1981).

6. P. Gotta et al., Phys. Lett. 112B, 129 (1982).

7. G. Backenstoss et al., Phys. Lett. 137B, 329 (1984).

8. M. A. Moinester et al., Phys. Rev. Lett. 52, 1203 (1984).

9. G. Backenstoss et al., Phys. Rev. Lett. 55, 2782 (1985).

10. K. A. Aniol et al., Phys, Rev. C33, 1714 (1986).

11. L. C. Smith, Ph.D. thesis, University of Virginia, 1987 (unpublished).

12. U. Sennhauser et al., SIN (PSI) Proposal R87-13.1 (1987).

13. C. S. Mishra et al., LAMPF Proposal No. 1071 (1987); R. Ransome et al., LAMPF Proposal No. 1143 (1988).

14. G. Lolos et al., TRIUMF Proposal No. 555 (1988).

15. S. A. Wood, Los Alamos National Laboratory report LA-9932-T, 1984 (unpublished).

16. S. A. Wood et al., Phys. Rev. Lett. 54, 635 (1985).

17. J. L. Matthews, in "Proceedings of the LAMPF Workshop on Pion Double Charge Exchange," Los Alamos National Laboratory report LA-10550-C (1985), p. 275.

18. E. R. Kinney et al., Phys. Rev. Lett. 57, 3152 (1986).

19. P. A. M. Gram, in Pion-Nucleus Physics: Future Directions and New Facilities at LAMPF, ed. by R. J. Peterson and D. D. Strottman, AIP Conf. Proc. 163, 79 (1988).

20. E. R. Kinney, Los Alamos National Laboratory report LA-11417-T, 1988 (unpublished).

21. P. A. M. Gram et al., Phys. Rev. Lett. 62, 1837 (1989).

22. J.-F. Germond and C. Wilkin, Lett. Nuovo Cim. 13, 605 (1975).

23. J.-B. Jeanneret, M. Bogdanski, and E. Jeannet, Nucl. Phys. A350, 345 (1980).

24. T. Takaki and M. Thies, Phys. Rev. C38, 2230 (1988).

25. S. Gilad et al., Phys. Rev. Lett. 57, 2637 (1986).

26. J. D. Silk et al., LAMPF Proposal No. 1120 (1988).

27. W. R. Gibbs, in "Proceedings: Physics with Light Mesons and the Second International Workshop on $\pi N$ Physics," Los Alamos National Laboratory report LA-11184-C (1987), p. 28.

28. J. R. Comfort, in Hadronic Probes and Nuclear Interactions, ed. by J. R. Comfort, W. R. Gibbs, and B. G. Ritchie, AIP Conf. Proc. 133, 242 (1985).

29. N. Grion et al., Nucl. Phys. A492, 509 (1989).

30. A. Rahav, private communication (1989).

31. A. Shor, Phys. Rev. Lett. 54, 122 (1985).

32. C. Morris and J. McGill, in "Progress at LAMPF 1986," Los Alamos National Laboratory report LA-11048-PR (1987), p. 211.

33. G. R. Smith, "CHAOS Design Note," 1989 (unpublished). 


\title{
MESON PRODUCTION
}

\author{
W. R. Gibbs, J.-C. Peng, and C. S. Mishra \\ Los Alamos National Laboratory \\ D. Isenhower \\ Abilene Christian University
}

\section{Recent Achievements}

Eta production has been studied at LAMPF with both exclusive and inclusive reactions.

The etas were observed in a number of different manners: by detecting the recoiling hadronic system (neutron or triton), or by measuring the eta directly using the pi-zero spectrometer, a simple BGO array, or a more sophisticated eta spectrometer composed of NaI with BGO.

The measurements of eta production on the proton give a value of the threshold production cross section.

The reaction on ${ }^{3} \mathrm{He}$ provided the first pion-induced exclusive eta-nucleus production cross section ever measured. Comparison of this reaction with theory shows a disagreement of about a factor of three in magnitude, although the shape of the angular distribution is well reproduced.

From the dependence of the inclusive cross section on mass number, the eta-nucleon reaction cross section has been estimated to be around $15 \mathrm{mb}$, which is about a factor of two smaller than the pion-nucleon cross section at the same momentum and in substantial agreement with the additive quark model.

Present limitations in beam energy restrict the measurements to threshold ( $680 \mathrm{MeV} / \mathrm{c})$ or subthreshold production. Even slight increments in the pion beam energy would be significant in terms of production rate.

\section{Eta Physics: An Eta Factory}

The eta meson holds a special place in the study of hadronic and weak interaction physics. Since it is a member of the light meson octet, its wave function is relatively simple. Still, it is unique, since all of the other mesons in this group have a quark-antiquark structure, while the eta is a mixture of strange and nonstrange $q \bar{q}$ pairs. The fraction of strange vs nonstrange components can be determined, as we will note below. The eta provides a laboratory to test our ideas about hadron structure and can itself be used as a probe of the structure of baryon resonances. This fundamental light meson has been inadequately explored and is among the most fertile of all the hadronic systems. In this respect it is interesting to note that its lifetime, which is very important for the absolute determination of its various partial widths, is known less well than that of the $B$ or $D$ mesons (20\%, compared to $10 \%$ for the $B^{0}$ meson and $2.5 \%$ for the $D^{0}$ meson).

One of the straightforward advantages of the eta comes from the fact that its significantly larger mass permits multiple pion decays. For example, the $\pi \pi \gamma$ decay can be treated by soft pion techniques, and the isospin-violating $3 \pi$ decay is sensitive to the difference in the up and down quark masses. 
For the weak decays, the larger mass of the eta means that the unitary limit from electromagnetic decay comes at a significantly lower value, opening a larger window for new physics. Here one also has the feature that the eta has an $s \bar{s}$ content, thus opening a new dimension in searching for new physics beyond the Standard Model.

One reaction of great interest to the quark model is the production of pairs of pi and efa mesons from proton targets. In this way the pi-eta scattering can be measured and compared with the quark-model prediction. ${ }^{1}$

\section{Tagged Beams of Etas}

With an $800-\mathrm{MeV} / \mathrm{c}$ beam of $10^{9} \pi / \mathrm{s}$, a $10-\mathrm{cm}$ liquid hydrogen target will produce $1.4 \times 10^{6} \mathrm{\eta} / \mathrm{s}$, or $10^{11} \eta /$ day. An efficient tagging system would allow the production of tagged etas with a flux about one order of magnitude smaller.

This work would complement a number of experiments planned at other facilities. At Saclay, with the $d+p \rightarrow{ }^{3} \mathrm{He}+\eta$ reaction, $10^{3}$ tagged etas per cycle can be obtained, and with improvements in the beam and system, $10^{5}$ can be reached. At KEK, similar rates are anticipated. In LEAR experiments at CERN, it has been suggested that one can attain untagged production rates of a few $\times 10^{5}$ particles $/ \mathrm{s}$ from antiproton annihilation for the measurement of $\eta, \eta^{\prime}, \rho$, and $\omega$ decays. ${ }^{2}$

An important advantage of the production of etas with pion beams, over that with proton or deuteron beams, is that the background is less by about four orders of magritude, permitting more accurate measurements and lower limits on rare decays.

There are a number of decays which address questions in current algebra, often revolving around the $\eta \cdot \eta^{\prime}$ mixing angle. ${ }^{3}$ One of the important ingredients in the extraction of this important quantity is the $\gamma \gamma$ decay width (current value $0.514 \pm 0.017 \pm 0.035 \mathrm{keV}$ ). The value of this width is ir.portant for the extraction of the light vs strange quark content of the eta. Note that there is a significant discrepancy in this width obtained from $\gamma \gamma \rightarrow \eta$ and the Primakoff effect. The later measurement gives $0.324 \pm \mathbf{0 . 0 4 6}$, which is about a three-sigma difference.

The soft pion prediction for $\eta \rightarrow \pi \pi \gamma$ is a factor of two below the experimentally observed width for this process.

Quark models predict the radiative branching ratios for rho compared to omega. The experimental number for $(\rho \rightarrow \pi \gamma) /(\omega \rightarrow \pi \gamma)$ is $0.093 \pm 0.015$, in agreement with the prediction of 0.105 . An improvement in the measurements involved in this ratio would permit a more accurate test. The ratio $(\rho \rightarrow \eta \gamma) /(\omega \rightarrow \pi \gamma)$ is a measure of the nonstrange quark content of the eta. The present value of this ratio $(0.072 \pm 0.019)$ leads to an amplitude for the probability for nonstrange quarks of $0.76 \pm 0.06$. The theories to be compared with these measurements in these cases are not extremely accurate at the present time, but it is shameful that the experimental determination of these fundamental values are not even good enough to test them.

The eta has very strong isospin-violating decays. A clear example is the decay into $\pi^{+} \pi^{-} \pi^{0}$. The width for this decay has been interpreted as a direct measure of the difference in the masses of the up and down quarks. The current value of this width $(250 \pm 36 \mathrm{eV})$ yields the ratio to the 
strange quark mass of $\left(m_{u}-m_{d}\right) /\left(2 m_{s}\right)=0.017 \pm 0.0012$ (or $0.014 \pm 0.004$, after some theoretical corrections).

We note that a major problem with these "strong" decay widths is that they must be known absolutely, since they are compared with other absolute widths in other mesons (e.g., $\pi^{\mathbf{0}}$ or $\eta^{\prime}$ ). Since a direct measure of the eta lifetime seems to be difficult, the widths are obtained from branching ratios and the absolute measurement of one of the widths. The large branching ratios are measured to the order of $1 \%$. At the present time, the $\eta \rightarrow \gamma \gamma$ reaction determines the accuracy of all of the rest.

For the rare decays this is not a problem. Using the number of etas estimated above, studies of bra 1 ching ratios of the order of $10^{-11}$ to $10^{-12}$ may be contemplated, allowing very low limits to be obtained. Since the eta production cross section at $800 \mathrm{MeV} / \mathrm{c}$ is $5 \%$ of the pion total cross section, the total interaction rate will be only twenty times the "good" events. For tagged eta production with proton beams, this ratio will be about $10^{5}$. The rare decays of the eta can be of special interest in certain cases. ${ }^{4,5}$

Two rare decays of particular interest are $\eta \rightarrow e^{+} e^{-}$and $\eta \rightarrow \pi^{+} \pi^{-}$. The present limits on the branching ratios of these two decays are about $3 \times 10^{-4}$ and $1.5 \times 10^{-3}$ respectively. The electromagnetic contribution to the first decay is expected to be seen at a level of about $10^{-8}$ to $10^{-9}$. This smaller size (about 20 times smaller than $\pi^{0} \rightarrow e^{+} e^{-}$) for this "background" (due to the larger mass of the eta) leaves a greater window for possible new physics to be uncovered.

The second decay offers a very exciting opportunity because it is CP-violating. Since CP violation has so far only been seen in the kaon system, its observation in a different reaction would add a new dimension to the field. Based on our current knowledge of the dipole moment of the neutron, it would be very surprising if the branching ratio were much above $10^{-15}$, which is well below what could be done in these experiments (or possibly any others).

Measurements of $\eta \rightarrow \mu e$ with a sensitivity to branching ratios of $10^{-9}$ io $10^{-10}$ would provide new information on possible muon-number-nonconserving interactions. The present indirectly determined limit is $8 \times 10^{-2}$. It is interesting to note that the limits for this decay are better known for the $D^{0}$ meson $\left(<1.2 \times 10^{-4}\right)$ than for the eta. Thus lepton-number violation is better studied in the charmed system than in the eta.

The measurement of muon polarization in $\eta \rightarrow \mu^{+} \mu^{-}$(branching ratio $6.5 \times 10^{-6}$ ) beyond the 0.1 level is a very interesting probe of possible CP-violating muon-quark neutral current interactions.

\section{Production and Decays of Higher Mass Mesons}

The production and decays of omega and rho mesons can be studied in free space with pion beams up to $1.2 \mathrm{GeV} / c$. As indicated by Adamus et al., ${ }^{6}$ there is still little known about the similarities or differences in the production of $\omega, \rho^{ \pm}$, and $\rho^{0}$ mesons, even though such knowledge is important for various parton models. This is mainly due to the difficulties in the identification and measurement of neutral pions, for which a good neutral meson spectrometer is the natural solution. Adamus et al. also point out that there is a discrepancy in the $\omega / \rho^{0}$ production ratio at $16 \mathrm{GeV} / c$ for $\pi p$ interactions. While none of the proposals for new capabilities at LAMPF will 
allow one to check these $16-\mathrm{GeV}$ results, lower-energy, high-intensity measurements will certainly be able to give a good measurement of the production ratio for omega to rho mesons.

Recent theoretical work ${ }^{7}$ has shown that, due to isospin breaking effects, $\rho-\omega$ mixing must be considered when the CVC hypothesis is used in order to relate the weak transition vector form factor to the corresponding electromagnetic one. This mixing also has direct interpretations in terms of up and down quark mass differences. Additional work on this topic with high intensity pion beams may be expected to give new and interesting results.

The mass difference between the $\rho^{ \pm}$and $\rho^{0}$ is presently consistent with zero (the Particle Data Group gives $\left.m\left(\rho^{0}\right)-m\left(\rho^{ \pm}\right)=0.3 \pm 2.2 \mathrm{MeV}\right)$, but the error on this result leaves room for a nonzero value. Further measurements should be able to reduce the error bars somewhat if one has good neutral pion detection capabilities.

There is one meson which is presently notable by its apparent absence. That is the scalar strange meson, the kappa. It should lie in an energy range just above $1 \mathrm{GeV}$ and would require higher-energy pion beams to search for it.

\section{Meson Production in Nuclei: Using the Nuclens as a Laboratory}

By coherent production and propagation through nuclei, the properties (structure and/or interactions with nucleons) of the $\delta, S^{*}, \phi, \eta^{\prime}$, etc., mesons can be studied. (The new names for the $\delta$ and $S^{*}$ in the Review of Particle Properties are $a_{0}$ and $f_{0}$.) In particular, there are techniques for investigating the possible molecular-like structure of the $S^{* 8}$

Production of rho mesons from nuclei may give the opportunity of the measurement of their masses and widths in the nuclear medium to determine if they have been altered from those in free space. Charged rhos with the same charge as the pion beam can be coherently produced (in the wave mechanical sense) from nuclei in an analogous fashion to the $\left(\gamma, \pi^{0}\right)$ reaction. The $\left(\pi^{+}, \rho^{-}\right)$ reaction gives the possibility of an exotic form of double charge exchange.

The production of eta mesons from polarized ${ }^{10} \mathrm{~B}$ will allow the study of the eta-nucleon interaction. The asymmetry in this case arises from the distortion effects alone; the distortion of the incoming pion has the opposite effect from that of the produced eta, so that it is the difference in the two interactions which is measured.

For the eta, for example, one can actually observe the production and subsequent reaction in the nucleus. Consider the production of etas by a pion beam followed by the interaction of the eta with a nucleon, leading to a pion with the opposite charge from that of the beam. By kinematical selection, those pions produced by the eta reaction leading to pions can be distinguished from those arising from sequential double charge exchange with an intermediate $\pi^{0}$. One knows the cross section for the $\eta N \rightarrow \pi N$ reaction from detailed balance, so this measurement can be used to check our understanding of the mechanism.

For high-energy double charge exchange to definite final states at high energy, the eta becomes a possible on-shell intermediate state. For this reason it becomes of interest to measure the exclusive single charge exchange. 
Another interesting channel is the interaction $\eta N \rightarrow \pi \pi N$. Due to the lack of "true" eta beams, this reaction is not directly measurable on a nucleon target. We note here the correlation with measurements on baryon resonances since the (perhaps dubious) $P_{13}$ state at $1545 \mathrm{MeV}$ was seen in eta production and $(\pi, 2 \pi)$ reactions, but not in pion scattering experiments. The $\eta N \rightarrow \pi \pi N$ reaction is another way to address the same hadronic physics in this region.

\section{References}

1. J. Weinstein, in "Proceedings of the LAMPF Workshop on Physics with Light Mesons," Los Alamos National Laboratory report LA-11184-C (1987), p. 10.

2. L. Bergstrom and H. R. Rubinstein, Phys. Lett. B203, 183 (1988).

3. F. J. Gilman and R. Kaufmann, Phys. Rev. D36, 2761 (1987).

4. P. Herzceg, in Production and Decay of Light Mesons, P. Fleury, Ed. (World Scientific, Singapore, 1988), p. 16.

5. A. Soni, Ref. 1, p. 55.

6. M. Adamus et al., Phys. Lett. B198, 292 (1987).

7. G. Castro and J. Pestieau, Phys. Lett. B203, 315 (1988).

8. W. R. Gibbs and W. B. Kaufmann, Ref. 1, p. 28.

9. P. B. Siegel and W. R. Gibbs, Phys. Rev. C36, 2473 (1987). 


\title{
$\left(\pi^{+}, K^{+}\right)$PHYSICS
}

\author{
B. F. Gibson and J. Cohen \\ Los Alamos National Laboratory \\ R. Chrien and J. Millener \\ Brookhaven National Laboratory \\ E. V, Hungerford \\ University of Houston
}

The strangeness degree of freedom (flavor) in hypernuclei adds a new dimension to our evolving picture of nuclear physics. First, the hyperon provides a tagged probe with which to explore the structure of the nuclear core states-differentiating probe interactions from those of target constitutents. Furthermore, hypernuclear structure exhibits qualitative differences from the structure of nonstrange systems. Second, embedding a strange quark in a sea of up- and down-quarks opens to question whether hyperons are such strongly bound (quark-gluon) systems that they are truly distinguishable Fermions, distinct from ne: tron and proton, or whether the hyperon substructure reveals itself when immersed in the nuclear medium. Finally, the nonmesonic weak decay of hypernuclei enables one to explore the $\Lambda N \rightarrow N N$ strangeness-changing weak interaction, which is inaccessible in free space and probes short distance phenomena characteristic of the weak force. Hypernuclear research opens new horizons in the field of strong and weak interaction many-body physics.

\section{Structure}

Coupling a $\Lambda$ to a nucleus can be used to modify the moment of inertia of a deformed nucleus, altering the rotational band structure, to produce an observable change in the phonon spectrum of a vibrational nucleus, or to shift the energy gap in a superfluid nucleus. ${ }^{1}$ Adding a $\Lambda$ to nuclei in mass regions that encompass oblate-to-prolate phase transitions will alter the mass value at which the transition occurs. ${ }^{2}$ Inserting a $\Lambda$ will influence the properties of shape isomers. Compression due to the binding of a $\Lambda$ will increase the Coulomb energy of the core nucleus. ${ }^{3}$ Adding a $\Lambda$ can raise the threshold for particle emission, making low-lying continuum states of the core stable against particle decay. ${ }^{4}$ As a nuclear structure probe the $\Lambda$ has many facets, but these intriguing possibilities remain totally unexplored.

Hypernuclei test our understanding of structure, constrain our models of nuclear forces, and exhibit new phenomena. The $\Lambda N$ spin-orbit force has been shown to be an order of magnitude smaller than the $N N$ but has yet to be precisely determined. ${ }^{5}$ A small spin-orbit force places a strong constraint on the relativistic $\sigma-\omega$ inodel, where the central force is closely (transparently in the QHD mean field limit) related. ${ }^{6}$ Because long range OPE is absent from the $\Lambda N$ interaction and $\Lambda N-\Sigma N$ coupling is strong, three-body $\Lambda N N$ forces are predicted to play a crucial role in hypernuclei-from being essential to the binding of the hypertriton to being strongly repulsive in the mass- 5 system and nuclear matter. ${ }^{7}$ Detailed shell-model calculations in the $p$-shell have revealed fascinating deviations from the weak coupling picture which highlight a new and unique feature of hypernuclear structure: Supersymmetries that are Pauli-forbidden in normal nuclei can play an important role. ${ }^{8}$ Exploiting hypernuclei to increase our understanding of nuclear structure 
requires significant improvement in resolution, to the order of $100 \mathrm{keV}$. Individual-level quantum numbers as well as positions must be measured.

\section{Quark Substructure}

The conventional hadron view holds the $\Lambda$ to be one of a multiplet of baryons, distinguishable from both proton and neutron, whereas QCD pictures the $\Lambda$ as a composite of up-, down-, and strange-quarks. How does one understand the Pauli principle in this case? In particular, one might anticipate that the $\Lambda$ would experience a Pauli pressure due to the other up- and down-quarks when one tries to embed a $\Lambda$ deep within the nucleus. ${ }^{9}$ Although present experimental results on the evolution with $A$ of ground- and excited-state energies are apparently consistent with a simple one-body (mean field) potential for the $\Lambda,{ }^{10}$ such quark substructure effects are clearly more subtle than any experiment presently envisioned would reveal. The formation of supersymmetric states confirms the tendency of hypernuclei to seek the higher level of spatial symmetry expected from a distinguishable $\Lambda$. Search for and identification of supersymmetric states in heavier systems is important. Good resolution is imperative if one is to extract the required level of information as a function of mass to address such questions. Finally, the najve quark model predicts the strength of the $\Sigma N$ spin-orbit force to be comparable to the $N N$ whereas boson exchange models predict it to be about $1 / 3$ the $N N .^{11}$ It is presently unknown experimentally.

\section{Weak Interaction}

The free $\Lambda$ decays principally via $\Lambda \rightarrow N \pi$, the nucleon having a momentum of some $100 \mathrm{MeV} / \mathrm{c}^{12}$ This mesonic decay is severely inhibited by Pauli blocking in all but the lightest hypernuclei, and its study would yield information about nuclear wave functions. ${ }^{13}$ For $A>5$ the major decay mode becomes the weak nonmesonic $\Lambda N \rightarrow N N$ reaction. ${ }^{14}$ Does the $\Delta I=1 / 2$ rule deduced from free hyperon decays still hold in the nucleus? ${ }^{15}$ One can explore the fourFermion, strangeness-changing weak interaction only in hypernuclei-there are no data on the free $\Lambda N \rightarrow N N$ reaction. ${ }^{16}$ The $\Lambda N \rho$ weak vertex can be investigated only by this means. Partial decay rates for the proton-stimulated $(p \Lambda \rightarrow p n)$ and neutron-stimulated $(n \Lambda \rightarrow n n)$ transitions are sensitive to the relative contribution of different meson exchanges $(\pi, \rho, K, \ldots)$ to the weak decay process. ${ }^{17}$ Detailed investigations of the nonmesonic weak decays of hypernuclei may also enable one to explore the short distances relevant to the quark nature of the baryons. ${ }^{18}$ Do these weak decays support the suggestion from the EMC effect analysis and $K^{+}$-nucleus scattering that baryon properties are modified in the medium ${ }^{19}$ Improved statistics (10\% uncertainties for partial rates and data for $A>12$ ) are essential to progress in this area.

\section{Accomplishments}

Hypernuclear studies at CERN and BNL have established that unique insight comes from exploring the strangeness dimension. First and most important, the $\left(K^{-}, \pi \gamma\right)$ measurements in heavier systems ${ }^{20}$ enabled one to deduce the size of the spin-dependent $\Lambda N$ matrix elements in the $p$-shell hypernuclei including the $\Lambda N$ spin-orbit force, ${ }^{5}$ although there are still unsettled questions about the precise parameterization. Also, supersymmetric states in ${ }^{13} \mathrm{C}$ and ${ }_{\Lambda}^{9} \mathrm{Be}$ were identified, and the observation of the $5 / 2^{+} \rightarrow 1 / 2^{+}$gamma transition in ${ }_{\Lambda}^{7} \mathrm{Li}$ confirmed that the low-lying $3^{+}(2.18 \mathrm{MeV})$ continuum state in ${ }^{6} \mathrm{Li}$ is siabilized against particle decay with the addition of a 
$\Lambda .^{21}$ Second, the complementarity of the $\left(K^{-}, \pi^{-}\right)$and $\left(\pi^{+}, K^{+}\right)$reactions is illustrated by the investigation of ${ }_{\Lambda}^{9} \mathrm{Be}^{22}$ The lower $3 / 2^{-}$state is barely excited by the forward angle $\left(\mathrm{K}^{-}, \pi^{-}\right)$ reaction, because the $\Delta L=0$ transition is forbidden by symmetry arguments. The $\Delta L=0$ strength lies in the two strongly excited peaks $(T=0,1)$ at 7 and $19 \mathrm{MeV}$. In contrast, the $\left(\pi^{+}, K^{+}\right)$reaction favors the excitation of the higher spin states in ${ }^{9} \mathrm{Be}$, the "supersymmetric" $3^{-}$ state near $B_{\Lambda} \simeq 4 \mathrm{MeV}$ and the $2^{+}, 3^{-}$, and $2^{+}$states with $B_{\Lambda}=2 \mathrm{MeV},-4 \mathrm{MeV}$, and $-12 \mathrm{MeV}$, respectively. Third, the $\left(\pi^{+}, K^{+}\right)$reaction was demonstrated at BNL to be the reaction of choice to access the deep lying hypernuclear states. ${ }^{23}$ The evolution of $\Lambda$ binding energies (ground and excited states) has been tracked as a function of mass number up to $A \simeq 90$. The $A$ dependence of level spacings provides a crucial constraint on the geometry of the $\Lambda$-nucleus potential and its well depth. ${ }^{10}$ Finally, nonmesonic decay of ${ }^{12} \mathrm{C}$ and ${ }_{\Lambda}^{5} \mathrm{He}$ has been studied recently at BNL. ${ }^{24}$ Pauli quenching of the mesonic decay process was confirmed. The unexpectedly large rate for the $n \Lambda \rightarrow n n$ decay compared to the $p \Lambda \rightarrow p n$ decay remains a puzzle that disagrees with available model calculations that enforce the empirical $\Delta I=1 / 2$ rule. ${ }^{25}$ Hypernuclear physics awaits the tools necessary to carry out the research program that clearly calls. Resolution is the key to advancing to new levels of understanding.

\section{The $(\pi, K)$ Role}

The principal mechanisms for producing hypernuclei are $\left(K^{-}, \pi\right)$ strangeness exchange and $\left(\pi^{+}, K\right)$ associated production. ${ }^{22}$ The lower cross sections characteristic of $\left(\pi^{+}, K^{+}\right)$production of light hypernuclei, compared to $\left(\mathrm{K}^{-}, \pi^{-}\right)$, have been more than compensated for by the higher pion fluxes available, compared to kaons, in BNL experiments. ${ }^{23}$ The small momentum transfer possible in $\left(K^{-}, \pi\right)$ has led physicists to focus on the limited set of "substitutional" states, in which a nucleon is replaced by a $\Lambda$ with the same orbital quantum numbers, preferentially populated by this $\Delta L=0$ reaction. ${ }^{26}$ In $(\pi, K)$ reactions one finds $q>350 \mathrm{MeV} / c$ at the maximum of the elementary cross section near an incident pion momentum of $1050 \mathrm{MeV} / c$. The formation of high spin states is therefore favored, but ground states and low-lying excited states are also populated. ${ }^{23}$ Whereas the $(K, \pi)$ reaction becomes less effective in populating bound states of hypernuclei as one moves beyond the $p$-shell, because the valence nucleons have increasingly higher angular momentum, the $(\pi, K)$ reaction has proved to be a powerful tool for selective production of hypernuclear states with a surface nucleon hole and a $\Lambda$ in even the deepest orbital up to $A=90 .^{10,22}$ High-resolution $(\pi, K)$ studies $(\sim 200 \mathrm{keV})$ will make significant spectroscopy feasible. The $j_{N}^{-1} \ell_{\Lambda}$ particle-hole states will define the $\Lambda$ single-particle binding encrgies, which determine the characteristics of the $\Lambda$-nucleus mean field potential. Fine structure due to coupling of the $\Lambda$ to different core states will constrain the $\Lambda N$ effective interaction. The small, but as yet not precisely measured, $\Lambda$ spin-orbit splitting can be deduced from $\ell_{\Lambda} \neq 0$ state studies. Coincidence $\left(\pi^{+}, K^{+} \gamma\right)$ resolving the ground state doublet in ${ }^{10} \mathrm{~B}$ would bear directly on the spin dependence of the fundamental $\Lambda N$ force. The nat ural polarization predicted for hypernuclei formed in the $(\pi, K)$ reaction should further elucidate the structure: generally cross sections for natural parity states drop with increasing angle while the polarization increases. ${ }^{27}$ This polarization may also enable one to investigate the magnetic monent of the $\Lambda$ in hypernuclei, addressing again the question of medium modifications. ${ }^{6}$

The long mean free path of the $1050-\mathrm{MeV} / \mathrm{c}$ incident $\pi^{+}$and the final state $\boldsymbol{K}^{+}$imply that initial- and final-state interaction effects will distort the analysis of $(\pi, K)$ reactions much less than that of the complementary $\left(K^{-}, \pi\right)$ reaction. Concomitantly, the $(\pi, K)$ reaction can populate 
more deeply bound states within the nucleus; that is, the reaction is less peripheral or surface confined. Thus, a clean pion beam with about $1 \mathrm{GeV} / c$ momentum should make an ideal tool for pursuing a forefront program of hypernuclear physics research. In order to undertake such a $(\pi, I)$ program one needs pion intensities of order $10^{9} / \mathrm{s}$, with missing-mass resolution of order $100 \mathrm{keV}$. This requires an improvement of a factor of $10^{2}$ in pion intensity and $\mathbf{3 0}$ in resolution. The beam should permit placement of secondary detectors (e.g. NaI, Ge, or wire chambers) near the target to observe in coincidence decays of hypernuclei. Detailed models of hypernuclear structure as well as reaction mechanisms and decay have been developed but, in the absence of precision data, remain to be tested.

\section{References}

1. H. Bando, M. Seki, and Y. Shono, Prog. Theor. Phys. 66, 2118 (1981).

2. H. Feshbach, AIP Conf. Proc. 33, 521 (1976).

3. B. F. Gibson, A. Goldberg, and M. S. Weiss, Phys. Rev. 181, 1486 (1969).

4. A. Gal, Adv. in Nucl. Phys. 8, 1 (1977).

5. W. Brueckner et al., Phys. Lett. 73B, 157 (1978); D. J. Millener, A. Gal, C. B. Dover, and R. H. Dalitz, Phys. Rev. C31, 499 (1985).

6. J. Boguta and S. Bohrmann, Phys. Lett. 102B, 93 (1981); J. Cohen and R. J. Furnstahl, Phys. Rev. C35, 2231 (1987).

7. A. R. Bodmer and Q. N. Usmani, Nucl. Phys. A477, 621 (1981); B. F. Gibson and D. R. Lehman, Phys. Rev. C37, 679 (1988); I. R. Afnan and B. F. Gibson, Phys. Rev. C (to be published).

8. E. H. Auerbach et al., Phys. Rev. Lett. 47, 1110 (1981); R. H. Dalitz and A. Gal, Ann. Phys. (NY) 131, 314 (1981).

9. E. V. Hungerford and L. C. Biedenharn, Phys. Lett. 142B, 232 (1984); B. F. Gibson, AIP Conf. Proc. 133, 390 (1985).

10. D. J. Millener, C. B. Dover, and A. Gal, Phys. Rev. C38, 2700 (1988).

11. H. J. Pirner, Phys. Lett. 85B, 190 (1979); J. V. Nobel, Phys. Lett. 89B, 325 (1980); A. Bouyssy, Phys. Lett. 99B, 305 (1981).

12. R. H. Dalitz, in Nuclear Physics, C. de Witt and V. Gillet, Eds. (Gordon \& Breach, New York, 1969), p. 701.

13. T. Motoba, K. Itonaga, and H. Bando, Nucl. Phys. A489, 683 (1988); K. Itonaga, T. Motoba, and H. Bando, Z. Phys. A330, 209 (1988); R. Mach et al., Z. Phys. A331, 89 (1988); E. Oset and L. L. Saceldo, Nucl. Phys. A443, 704 (1983).

14. J. P. Lagnaux et al., Nucl. Phys. 60, 97 (1964); A. Montwill et al., Nucl. Phys. A234, 413 (1974); R. Grace et al., Phys. Rev. Lett. 55, 1055 (1985); J. Szymanski, AIP Conf. Proc. 150, 934 (1986); P. D. Barnes, Nucl. Phys. A478, 127c (1988).

15. B. H. J. McKellar, in Proceedings of the 1986 International Symposium on Hypernuclear Physics, H. Bando, O. Hashimoto, and K. Ogawa, Eds. (Univ. Tokyo, 1986), p. 146.

16. B. H. J. McKellar and B. F. Gibson, Phys. Rev. C30, 322 (1984).

17. J. F. Dubach, Nucl. Phys. A450, 71c (1986); J. F. Dubach, AIP Conf. Proc. 150, 946 (1986).

18. D. P. Heddle and L. S. Kisslinger, Phys. Rev. C33, 608 (1985).

19. P. B. Siegel, W. B. Kaufmann, and W. R. Gibbs, Phys. Rev. C31, 2184 (1985).

20. M. May et al., Phys. Rev. Lett. 47, 1106 (1981). 
21. M. May et al., Phys. Rev. Lett. 51, 2085 (1983).

22. E. C. Milner et al., Phys. Rev. Lett. 54, 1237 (1985); C. B. Dover, L. Ludeking, and G. E. Walker, Phys. Rev. C22, 2073 (1980); H. Bando and T. Motoba, Prog. Theor. Phys. 76, 1321 (1986).

23. J. C. Peng, AIP Conf. Proc. 176, 39 (1988); R. E. Chrien, Nucl. Phys. A478, 705c (1988); R. Hausmann and W. Weise, Nucl. Phys, A491, 598 (1989).

24. J. J. Szymanski, Ph.D. Dissertation (Carnegie Mellon Univ., 1987); P. D. Barnes, Nucl. Phys. A479, 89c (1988).

25. B. F. Gibson, in "Proceedings of the 1988 International Symposium on Hypernuclei and LowEnergy Kaon Physics," T. Bressani, Ed., Nuovo Cim. (to be published).

26. W. Brueckner et al., Phys. Lett. 55B, 107 (1975); W. Brueckner et al., Phys. Lett. 79B, 157 (1978); R. Bertini et al., Phys. Lett. 83B, 306 (1979); R. Bertini et al., Phys. Lett. 90B, 375 (1980); B. Povh, Ann. Rev. Nucl. Part. Sei. 28, 1 (1978); R. Bertini et al., Nucl. Phys. A477, 621 (1988).

27. H. Bando, T. Motoba, M. Sotona, and J. Zofka, Phys. Rev. C39, 587 (1989); H. Ejiri, T. Fukuda, T. Shibata, H. Bando, and K.-I. Kubo, Phys. Rev. C36, 1435 (1987). 


\author{
J. L. Matthews \\ Massachuset ts Instit ute of Technology \\ W. R. Gibbs and M. B. Johnson \\ Los Alamos National Laboratory \\ R. J. Peterson \\ University of Colorado
}

A. Pion single-charge-exchange $\left[\left(\pi^{+}, \pi^{0}\right),\left(\pi^{-}, \pi^{0}\right)\right]$ reactions (SCX) have comprised one of the most fruitful avenues pursued by LAMPF in pion-nucleon and pion-nucleus physics, and one in which LAMPF has made a unique contribution. Even with the modest (by nuclear-structure standards) resolution of $2-4 \mathrm{MeV}$ of the LAMPF $\pi^{0}$ spectrometer, there have been many notable accomplishments. For example, one might cite the following:

1) Measurements of the $\mathrm{SCX}$ cross section $\left(\pi^{-}+p \rightarrow \pi^{0}+n\right)$ verified that the phase-shift analysis of pion-proton elastic scattering performed by Arndt which produced a double-pole structure in the $N^{*}$ resonance region was correct. ${ }^{1}$

2) An isovector monopole excitation in the $\left(\pi^{-}, \pi^{0}\right)$ reaction on a range of nuclei was discovered and characterized. ${ }^{2}$

3) Investigation of the systematics of SCX to the isobaric analog state has begun, with zero-degree cross sections measured as a function of incident pion energy for several nuclei and angular distributions for a few favorable cases. ${ }^{3}$ This work has led to substantial new insights into the pion-nucleus interaction, in particular the modification of the elementary SCX amplitude in the nuclear medium.

4) The zero-degree SCX cross section to isobaric analog states in nuclei was discovered to have a deep minimum at 50-65 MeV, indicating an apparent transparency of the nucleus to pions at these energies. ${ }^{4}$

5) In a coincidence measurement, the quasifree charge-exchange process $\left(\pi^{+}, \pi^{0} p\right)$ has been studied for ${ }^{3} \mathrm{He}$ and ${ }^{16} \mathrm{O}$. The latter data, ${ }^{5}$ in comparison with those for the noncharge-exchange processes $\left(\pi^{+}, \pi^{+} p\right)$ and $\left(\pi^{-}, \pi^{-} p\right)$, provide strong evidence for the interaction of the $\Delta$ in the nucleus.

6) The production of $\eta$ mesons from nuclear targets has been observed. The first measurement of the exclusive $(\pi, \eta)$ process,${ }^{6}$ together with inclusive measurements, was used to estimate the $\eta$-nucleon reaction cross section.

7) Very recently, studies using aligned and polarized nuclear targets have begun. SCX on an aligned ${ }^{165} \mathrm{Ho}$ target was observed $;^{7}$ the asymmetry of the cross section with respect to target orientation will provide insight into the deformation of neutrons relative to protons in a heavy nucleus. The spin sensitivity of the pion-nucleon interaction in the nuclear medium is currently being invesíigated by means of SCX on a polarized ${ }^{13} \mathrm{C}$ target. ${ }^{8}$ 
B. A new neutron-meson spectrometer has been proposed which will have a factor of ten better resolution and a factor of two larger solid angular acceptance. A conceptual design study has been carried out and is being evaluated in detail. Two proposals for specific experiments have been approved by the LAMPF Program Advisory Committee; several others are in preparation. Research and development work for this instrument have begun at LAMPF and will continue through FY1990. The major technical issues to be resolved are the accuracy of vertex reconstruction possible with the proposed tracking chambers and the accuracy of the calorimetry achievable with the proposed material, pure CsI. As soon as it has been demonstrated that the design specifications can be met, a proposal for construction of the instrument will be submitted to DOE, which will also define the commitments of several user institutions that have expressed interest in participating in this project.

A draft of this proposal, which contains a detailed exposition of the physics justification and a discussion of current design ideas, will receive further work this summer and autumn. It is expected that this document will be available to interested users and to the LAMPF Program Advisory Committee for discussion at its January 1990 meeting.

C. The following is a brief summary of some of the physics questions that can be addressed by this instrument:

1) Fundamental processes: $\left(\pi^{-}, \pi^{0}\right)$ on unpolarized and polarized protons at low energies; extraction of the $\sigma$ term. ${ }^{9}$ There are no data for SCX on polarized protons below $192 \mathrm{MeV}$.

2) $\Delta$ 's in nuclei: the $\left(\pi^{+}, \pi^{0} p\right)$ reaction, to study the pion-nucleon interaction within the nucleus and to isolate intermediate $\Delta$-nucleon scattering.

a) At resonance energies, the $\left(\pi^{+}, \pi^{0} p\right)$ reaction is sensitive to the destructive interference between the quasifree knockout (impulse approximation) mechanism and that in which a $\Delta$ is produced which then knocks out a nucleon. ${ }^{10}$ In the $\left(\pi^{+}, \pi^{+} p\right)$ reaction constructive interference occurs, yielding less sensitivity. Good resolution for both the $\pi^{\mathbf{0}}$ and the proton is essential in order to identify the final nuclear states.

b) The resolution and efficiency of the NMS will allow the measurement of isovector spin excitations. The details of the excitation functions will establish the effect of the nuclear environment on the intermediate $\Delta$ selected by the nuclear quantum numbers.

c) With improved resolution, one can reliably use polarized nuclear targets to determine the pion-nuclear spin-flip amplitudes, providing clear access to the role of the $\Delta$.

3) Nuclear structure and reaction mechanisms. Study of $\left(\pi, \pi^{0}\right)$ in comparison with $\left(\pi, \pi^{\prime}\right)$ will allow at least partial disentangling of reaction mechanism and nuclear-structure effects. ${ }^{11}$ An additional handle on this separation is provided by exploiting the differences between the nucleon and pion probes. E.g., pion charge exchange (initiated by an $S=0, T=1$ particle) and nucleon charge exchange (initiated by an $S=1 / 2, T=1 / 2$ particle) provide complementary means of studying spin and isospin excitations in nuclei.

a) The pion single-charge-exchange ( $\mathrm{SCX}$ ) reaction exhibits a very special selection rule which is advantageous for nuclear-structure investigations. In the same way that (starting witlı 
a spin-zero, isospin-zero target) the $\alpha$-particle can excite only isospin-zero, natural-parity states, SCX at zero degrees can excite only isospin-one, natural parity states. Unlike the $\left(\alpha, \alpha^{\prime}\right)$ reaction, the SCX process may easily be observed at zero degrees. The angular distribution of transitions to both natural and unnatural parity states, after their initial identification, can then be followed over an extended angular range.

b) SCX using pions in the delta resonance region allows the neutron-proton transition densities in the extreme surface region of the nucleus to be measured, providing a test of the neutron wave functions predicted, e.g., by Hartree-Fock calculations. Testing these calculations for neutrons is particularly important since they are used to predict the properties of neutron stars. Aligned heavy nuclear targets will be of particular utility here.

c) With the improved resolution and efficiency of the NMS, cross sections for SCX to analog states could be measured out to large angles, determining the isovector part of the pion optical potential, in order to complete the characterization of this potential and help understand the corresponding medium modification of the pion-nucleon amplitude due to the presence of other nucleons. Many other nuclear studies rely upon pion optical potentials.

d) With the capability of making SCX measurements with good resolution, one can study the intermediate states through which pion double charge exchange (DCX) proceeds, and thereby separate nuclear-medium and nuclear-structure effects. E.g., the cross sections for ${ }^{48} \mathrm{Ca}\left(\pi^{+}, \pi^{0}\right)^{48} \mathrm{Sc}$ and ${ }^{48} \mathrm{Ti}\left(\pi^{-}, \pi^{0}\right)^{48} \mathrm{Sc}$ can both be measured, ${ }^{12}$ which will elucidate the DCX process ${ }^{48} \mathrm{Ca}\left(\pi^{+}, \pi^{-}\right)^{48} \mathrm{Ti}$. (It must be recognized that the contributions of intermediate states in DCX can have large off-shell components. These may be correlated with the results of the SCX measurements through generalizations of theoretical methods used at the present time, such as coupled channel techniques.)

e) Mediurn modifications of the isovector spin-flip amplitude can be studied by use of polarized nuclear targets. ${ }^{8,13,14}$ Transitions to nonanalog states, in particular spin-flip states, will require the improved resolution of the NMS.

4) $\eta$ 's in nuclei. ${ }^{15-18}$ The $\eta$, with mass $549 \mathrm{MeV}$, is the neutral isospin-zero partner of the isospinone $\pi^{0}$. Comparison of $(\pi, \eta)$ and $\left(\pi, \pi^{0}\right)$ reactions thus provides a means of studying the $\Delta$ and $N^{*}$ resonances in nuclei.

a) The NMS will permit investigation of $(\pi, \eta)$ reactions to resolved states in nuclei. One expects that these reactions will differ from the corresponding $\left(\pi, \pi^{0}\right)$ processes: since the $\pi^{ \pm}$and $\pi^{0}$ belong to the same isospin multiplet, these SCX processes are closely related to pion elastic scattering. The $(\pi, \eta)$ reaction involves a pair of particles with different isospin. Moreover, the momentum transfer in the $(\pi, \eta)$ reaction $\left(\sim 250 \mathrm{MeV} / c\right.$ at $\left.0^{\circ}\right)$ is large compared to that in the $\left(\pi, \pi^{0}\right)$ reaction; thus the former process may preferentially excite high-spin states.

b) At high energies, the DCX process may proceed through an intermediate $\eta$ state; with the NMS one could study the SCX $[(\pi, \eta)]$ reactions of interest. 
c) The basic processes of pionic $\eta$ production in the threshold region, in particular $\eta$ production on a neutron, can be investigated by observing $\pi^{+}+d \rightarrow \eta+p+p$ and $\pi^{-}+d \rightarrow \eta+n+n$ at $T_{\pi} \simeq 700 \mathrm{MeV}$. From these measurements one can determine the coupling constants and interaction ranges of the $\eta N N^{*}$ and $\pi N N^{*}$ vertices for the $N^{*}$ resonances. This information will be an important ingredient in future studies of $\eta$ mesons in a many-body nuclear environment, such as in relativistic heavy-ion collisions.

\section{References}

1. G. J. Kim et al., Phys. Lett. B218, 62 (1989).

2. A. Erell et al., Phys. Rev. C34, 1822 (1986); F. Irom et al., Phys. Rev. C34, 2231 (1986).

3. A. Doron et al., Phys. Rev. C26, 189 (1982).

4. F. Irom et al., Phys. Rev. Lett. 55, 1862 (1985).

5. S. Gilad et al., Phys. Rev. Lett. 57, 2637 (1986); S. Hoibraten, Los Alamos National Laboratory report LA-11582-T, 1989 (unpublished).

6. J.-C. Peng et al., Phys. Rev. Lett. 58, 2027 (1987).

7. J. N. Knudson et al., Phys. Rev. C35, 1382 (1987).

8. J. Comfort et al., LAMPF Proposal No. 1023 (1986).

9. See, e.g., J. Gasser, in "Proceedings: Physics with Light Mesons and the Second International Workshop on $\pi N$ Physics," Los Alamos National Laboratory report LA-11184-C (1986), p. 266.

10. T. Takaki and M. Thies, Phys. Rev. C38, 2230 (1988).

11. M. B. Johnson and M. K. Singham, in "Proceedings of the LAMPF Workshop on Photon and Neutral Meson Physics at Intermediate Energies," Los Alamos National Laboratory report 11177-C (1987), p. 275.

12. C. F. Moore et al., LAMPF Proposal No. 1167 (1989).

13. D. J. Ernst, in "Proceedings of the LAMPF Workshop on Physics with Polarized Nuclear Targets," Los Alamos National Laboratory report LA-10772-C (1986), p. 129.

14. P. B. Siegel and W. R. Gibbs, op. cit., p. 138.

15. M. G. Huber and B. C. Metsch, in "Proceedings: Physics with Light Mesons and the Second International Workshop on $\pi N$ Physics," Los Alamos National Laboratory report LA-11184-C (1987), p. 91.

16. L. C. Liu, op. cit., p. 104.

17. P. B. Siegel and W. R. Gibbs, Phys. Rev. C36, 2473 (1987).

18. J.-C. Peng, in Production and Decay of Light Mesons, P. Fleury, Ed. (World Scientific, Singapore, 1988), p. 102. 


\section{EXCERPT FROM}

\section{THE USER'S VIEW FOR THE FUTURE OF LAMPF (JUNE 1989)}

\section{PION PHYSICS}

In this section we give a brief general overview of pion physics, respond to the NSAC questions in some detail, and present a brief summary of the current status and future needs of the field.

1. The Pion as a Nuclear Probe The nucleus is more than just an assembly of neutrons and protons, as it appears when studied at low energy. At very high energies, for example, the nucleons (and the nucleus) have been shown to have a quark substructure. At intermediate energies another type of substructure is more relevant. Here, the nucleon structure is expressed as excited states that decay largely by radiating mesons, making meson- and nucleon-excited states important degrees of freedom of the nucleus. For energies up to about $1 \mathrm{GeV}$, the baryons are limited to those with the lowest masses, the $\pi, \rho$, and $\omega$ mesons, and the $\Delta(1232)$ and $N^{*}(1440)$ resonances. Among the mesons, the pion plays a crucial role in strong-interaction physics, in that, as the lightest-mass strongly interacting boson, it governs the long-range interaction $(>1.5 \mathrm{fm})$ between hadrons. The pion is also believed to be a direct manifestation of the chirally-symmetric nature of the QCD Lagrangian; such a Lagrangian may prove to be the link between QCD and hadron structure and interactions. It is therefore a very special nuclear probe.

2. The Interactions of Pions in Nuclei The isospin-one pion exists in three charge states, so that both single- and double-charge-exchange reactions can take place. These reaction channels have analogies in the normal elastic, inelastic and quasi-elastic scattering, and in the excitation of giant resonances. Since the pion is a boson, it can be absorbed and produced in nuclei, it can produce other mesons and create nucleon resonances in nuclei, and even though it has no spin itself, it has spin-dependent nuclear and nucleon reäctions. No other commonly used nuclear probe exhibits such a range of interactions.

The most prominent feature of the pion-nucleus interaction is the $\Delta(1232)$ resonance, which is strongly excited for pion energies between 100 and $300 \mathrm{MeV}$. Here the pion-nucleon interaction is so strong that the nucleus appears black and exclusive pion reactions are restricted to the nuclear surface. At other energies, the pion mean free path is longer, and the pion probes more of the nuclear interior. The isospin-3/2 nature of the Delta resonance causes $\pi^{+} p$ and $\pi^{-} n$ interactions to be much stronger than $\pi^{+} n$ and $\pi^{-} p$. These simple features have led to some interesting discoveries in nuclear structure.

At higher energies, in the region of the $N^{*}$ resonances, two more qualtitative features useful for the study of nuclear structure appear: the relative sensitivity of $\pi^{ \pm}$to protons and neutrons is reversed from that at the Delta, and the pion can be used to add strangeness to the nucleus by the production of hypernuclei with the $(\pi, K)$ reaction.

3. What Has Been Accomplished in Studies of Pion Physics Extensive studies of pionnucleus interactions began about fifteen years ago, after the beginning of operation of LAMPF and the other meson factories with their high-intensity beams and specialized detector systems. 
New and important features of these reactions have been uncovered which have given deeper insight into the mechanisms involved and into nuclear structure. Our general understanding of the pion-nucleus and pion-nucleon interactions have undergone significant development and modification. There have been a number of surprises.

Some selected examples of this work include the following:

The first complete data set in the $\pi-N$ system was measured in the region of 307 to $625 \mathrm{MeV} / \mathrm{c}$. These measurements test models of this important energy region, including the hypotheses that the Roper resonance has two poles and that it is a hybrid (one-gluon) state. An example of these data, the asymmetry in pion charge exchange, is shown in Fig. 1. It is clear that they distinguish between the phase-shift solutions, preferring the one (by Arndt et al.) that contains two poles in the vicinity of the Roper resonance.

The inclusion of a correlation in the relative nucleon-nucleon wave function was found to be essential for the understanding of pion double charge exchange to analog states. At least an order of magnitude difference is seen at low energy in comparisons with calculations of cross sections using uncorrelated wave functions, both in the absolute magnitude and in the isotopic ratios within the same shell. Figure 2 shows the measurements made on ${ }^{14} \mathrm{C}$ at $50 \mathrm{MeV}$ compared with a calculation based on simple product wave function for the two valence nucleons. The band represents several calculations that include various degrees of correlation arising from simple shellmodel configurations and variations in the calculational techniques.

The isovector monopole giant resonance was observed for the first time. Figure 3 displays the difference of spectra taken at two different angles, showing that the resonance stands out clearly in the basic data. The dependence on nuclear mass of the excitation energy and width was studied with both the $\left(\pi^{-}, \pi^{0}\right)$ and $\left(\pi^{+}, \pi^{0}\right)$ reactions. While properties of this state are in agreenent with the theoretical expectations for the $\left(\pi^{-}, \pi^{0}\right)$ reaction, where the final state lies at about $20 \mathrm{MeV}$ excitation energy, the observed width and excitation energy are smaller than predicted for the $\left(\pi^{+}, \pi^{0}\right)$ reaction, where the final state lies at about $35 \mathrm{MeV}$ excitation. The isovector quadrupole state is not seen at the level of an order of magnitude below the calculated cross section.

Exotic collective nuclear states have also been identified for the first time in double-chargeexchange scattering. Predictions of such states built upon intrinsic nuclear excitations have been verified, with the observation of giant dipole resonances built upon the isobaric analog states and upon the giant dipole resonance itself. Spectra showing these states for $\left(\pi^{+}, \pi^{-}\right)$on ${ }^{93} \mathrm{Nb}$ are shown in Fig. 4.

4. Prospects for Future Research In order to move toward some kind of global understanding of the pion-nucleus interaction, much more work is necessary. Questions raised by the results obtained so far need further investigation. For certain areas, programs of study are clearly defined. In the near future, work is planned on single-charge-exchange scattering to nonanalog states, eta production, pion interactions with polarized nuclear targets, and additional double-charge-exchange scattering. For the far future (5-10 years), studies of hypernuclei, the production and decay of higher-mass mesons, the characteristics of higher nucleon resonances, and pion-nucleus scattering in the region of $N^{*}$ s are contemplated. 
To achieve these goals, the experimental manpower must be maintained at least at its current level and additional instrumentation is needed. An increase in the manpower involved in theoretical work is also needed in order to help achieve a better understanding of the results obtained so far and to provide guidance to future experimental work.

5. Responses to the NSAC Questions on Pion Physics The following responses to the NSAC questions were developed by the LAMPF pion community over a period of two months. During this time, six papers summarizing different aspects of pion physics were prepared as background by members of this community, and three working meetings were held at LAMPF which were attended by up to forty physicists, where these papers and questions were discussed.

\section{a. WHAT ARE THE IMPOR IANT ACCOMPLISHMENTS OVER THE LAST FIVE YEARS? SPECIFIC EXAMPLES?}

Because of the richness and diversity of this field, the list of important accomplishments over the last five years is long. We highlight some of the more important items here and provide a more

detailed list in the Appendix [to this excerpt]. Work at laboratorjes other than LAMPF is also included, in order to present an overall picture.

In general, we have achieved a much deeper understanding of pion-nucleon interactions, pion propagation in nuclei, and nuclear structure.

Worldwide measurements on low-energy pion-nucleon scattering have produced data that bear crucially on the problem of chiral symmetry breaking, while studies at the highest pion energies available have verified analyses incorporating a split structure of the $N^{*}(1440)$ and apparently exclude the hypothesis that it corresponds to the first excited state of the gluonic field.

With evidence for multinucleon pion absorption, the separation of long- and short-range mechanisms in pion double-charge-exchange scattering, the observation of an apparent transparency of the nucleus to low-energy pions, the identification of unexpected features in inclusive pion double charge exchange, and the observation of the first pion-induced eta meson production to a definite final nuclear state, significant steps have been taken in increasing our understanding of hadronic reactions.

Our knowledge of nuclear structure has been advanced through the discovery of the isovector monopole state and of other giant resonances built on lower states, by the determination of many proton and neutron matrix elements, and by the observation of the interior shell structure of a heavy hypernucleus with $(\pi, K)$ reactions, in which a neutron is replaced by a Lambda.

\section{b. WHAT WILL BE THE THRUST OF THE PHYSICS OVER THE NEXT FIVE YEARS?}

Some of the following will involve the use of new or expanded experimental facilities at LAMPF:

Measurements of single-charge-exchange scattering in nuclei, to investigate spin and isospin excitations in a reaction that is complementary to nucleon charge exchange, to study the intermediate states through which double charge exchange proceeds, and to obtain information on neutron densities in the nuclear surface, will be made possible through the construction of a proposed new high-resolution neutral-meson spectrometer. 
Further studies of eta production to resolved states in nuclei will provide a new probe of nuclear structure, while studies of the production of eta mesons on nucleons will further investigation of the $N^{*}(1440)$ and determine the $\eta N N^{*}$ and $\pi N N^{*}$ coupling constants. The scope of this work would be greatly enhanced by the addition of a small superconducting pion accelerator to the high energy pion $\left(P^{3}\right)$ channel.

Studies of various pion interactions with polarized nuclear targets will give information on the spin dependence of the Delta-nucleus interaction, on the structure of excited nuclear states, on pion-nucleus reaction mechanisins, and on the modification of the pion-nucleon spin-dependent interaction in the nuclear medium.

Studies of double-charge-exchange scattering to discrete final states will give additional information to help establish the relative importance of various mechanisms, such as sequential processes, interactions with meson clouds, and Delta-nucleon interactions.

c. WHAT WILL BE THE SINGLE MOST IMPORTANT PIECE OF PHYSICS TO ACCOMPLISHED OVER THE NEXT FIVE YEARS?

We fully expect to make significant progress in completing and understanding the systematics of pion-nucleus interactions, from both an experimental and a theoretical point of view, and in refining our understanding of the pion-nucleon interaction, at energies up to about $500 \mathrm{MeV}$.

\section{d. ARE THERE COMPLETELY NEW AREAS OPENING UP OVER THAT PERIOD?}

Measurements of single-charge-exchange scattering to discrete nuclear states using a new highresolution neutral-meson spectrometer will allow for careful studies of isovector transitions in nuclei.

Measurements of pion interactions with polarized nuclear targets will permit detailed investigation of spin-dependent amplitudes.

\section{e. ADEQUACY OF FACILITY OR NEED FOR NEW INSTRUMENTATION FOR THE NEXT FIVE YEARS?}

The following are needed to maintain the vitality of this field:

The full-capacity operation of LAMPF, including all pion beam lines and detector facilities, must be continued. Any reduction would greatly hinder the progress of pion physics.

Perhaps the most important new facility needed at LAMPF in the next five years is the proposed high-resolution neutral-meson spectrometer. It will open up a new field of research, that of investigating nonanalog single charge exchange scattering. It will also allow the continuation of studies of eta production in nuclei and on nucleons and additional studies of quasifree charge exchange scattering to further investigate the interactions of Deltas in nuclei.

The upgrade of the Low Energy Pion channel, now in progress, which involves the addition of the superconducting energy compressor, or "SCRUNCHER," is very important. It will allow for higher intensity within a small momentum bite and is expected to give an increased data rate at low energies of a factor of up to five. 
A small superconducting pion accelerator on the $\mathrm{P}^{3}$ channel would be very desirable. With 50-100 MeV additional energy, the maximum energy of that channel would extend comfortably above the eta production threshold and increase the eta flux by an order of magnitude, as well as provide increased beam flux for additional studies in the energy region of the $N^{*}(1440)$.

Continued support and development of capabilities for the use of polarized nuclear targets is vital if these studies are to proceed in a timely manner.

\section{f. SPECIFIC PROJECTS OR INITIATIVES FOR SMALLER GROUPS?}

Most of the pion work at LAMPF has been carried out by collaborations among small groups. Important new instrumentation has also been developed with collaborations between LAMPF staff and small groups. This style of operation has proved to be very effective, and we expect it to continue.

\section{g. WHAT WILL BE THE DEVELOPMENT FOR THE FAR FUTURE (TEN YEARS)?}

The major new facility needed for pion physics in this time frame is a high-intensity pion beam of energy about $1 \mathrm{GeV}$ with appropriate detector facilities, including the capacity for highresolution studies, as described in the response to question $h$ below. This would allow all of the following programs to be pursued at a good pace:

High-resolution studies of hypernuclei. I'roduction of hypernuclei through the $\left(\pi^{+}, K^{+}\right)$reaction is complementary to studies using the $\left(\mathrm{K}^{-}, \pi^{-}\right)$process and is far superior for many purposes. The small momentum transfer of the latter reaction leads principally to a limited set of neutronLambda substitutional states, whereas the former favors the formation of high-spin states as well as low-lying and ground states. For high-resolution studies of the spectroscopy of hypernuclei, $(\pi, K)$ is the reaction of choice, and the existence of a high-flux high-resolution pion beam at $1.05 \mathrm{GeV} / \mathrm{c}$ would make such studies possible for the first time. Among the questions that can be investigated are whether the Pauli principle applies more properly at the nucleon level or the quark level in hypernuclei and the $\Lambda N \rightarrow N N$ strangeness-changing weak interaction, which is inaccessible in free space.

The production and decay of $\eta, \rho$ and $\omega$ mesons. A new dimension in the investigation of mesons in free space and in nuclei is opened by the existence of an intense high-energy pion beam. The modification of the properties of these mesons in the nuclear medium and their reaciions with nucleons can be studied, as well as many of their intrinsic properties as seen through rare decays, branching ratios, and mass differences.

Studies of double charge exchange at high energies. Around $1 \mathrm{GeV}$, the zero-degree doublecharge-exchange cross section is predicted to decrease sharply if the process is assumed to proceed by sequential single charge exchanges. This feature provides an excellent opportunity to search for suggested new interaction mechanisms, such as pion interactions with meson clouds or multiquark configurations, which would be revealed by the mimimum in the normally large background process.

Studies of higher mass nucleon resonances. A $1.2-\mathrm{GeV} / c$ pion beam will allow studies of the low-lying $D_{13}$ and $S_{11}$ resonances, as well as the cluster of six $N^{*}$ resonances in the 1.6-1.7 GeV mass range, which appear to be degenerate and whose existence may be related to meson production 
thresholds. Data with polarized proton targets as well as measurements of inelasticities will be needed.

Measurements of pion-nucleus scattering in the region of the $N^{*}$ resonances. Here, the relative sensitivity of $\pi^{+} / \pi^{-}$to protons and neutrons is reversed from that at the Delta resonance energy. One possible effect of $N^{*}$ s may be the strikingly different behavior of elastic angular distributions that has been observed at 500 and $660 \mathrm{MeV}$.

\section{h. WHAT IS THE MAJOR NEW FACILITY NEEDED IN YOUR AREA (IF ANY) IN THE} NEXT TEN YEARS?

In order to continue research in pion physics as discussed in the response to question $\mathrm{g}$ above, a pion beam is needed with momenta up to $1.2 \mathrm{GeV} / \mathrm{c}$, with a flux of $10^{9} \pi^{+}$per second over $1 \%$ $\Delta p / p$, and with suitable detectors, including the capacity for high-resolution studies. This flux will produce more than 100 counts per day, for example, in exclusive reactions for hypernuclear formation and pion double charge exchange.

\section{i. WHAT IS THE MANPOWER SITUATION IN YOUR AREA?}

For experimental work, the current level of manpower is marginally adequate. For the future, it appears to be deteriorating, partly due to the shortage of positions available for new research scientists.

For theoretical work, the situation is worse, again due to the shortage of available positions.

\section{j. FUNDING SITUATION IN YOUR AREA, NOW AND FUTURE NEEDS?}

The current level of funding for pion research at LAMPF is barely adequate to continue the current level of research. Possible cuts that would lead to reduced beam time or facility operation would be disastrous for the pion physics programs. We note that the three LAMPF pion channels are unique: the Low Energy Pion channel has the greatest brightness available for low-energy studies, the EPICS system is unmatched anywhere for high-resolution studies in the Delta region, and the $\mathrm{P}^{3}$ channel is the only place in the world where good-resolution studies can be carried out at energies above the Delta resonance.

For the next ten-year period, the pressing need is for a high-intensity pion beam of energy up to about $1 \mathrm{GeV}$, as described above. Substantial funding will be needed for this project.

The level of funding for theoretical work is low enough to be a cause of great concern. Highly qualified young people are being forced to leave the field because of a lack of positions. This is particularly detrimental for the pion physics program in its present state of development, in which interaction between experiment and theory serves as an important stimulation to indicate useful directions for future work.

6. Summary Pion physics has been, and continues to be, a broadly-based field of vital and productive research which has given important new insights into nuclear and hadronic structure. Given sufficient resources, this vitality and productivity will continue, and this research will expand into new fields of study. 
The needed resources on a 0 - to 5 -year time scale include continued support of pion physics at LAMPF at the current level, the construction of a new high-resolution neutral-meson spectrometer, and increased support for theoretical work.

Over a 5- to 10-year time frame the creation of a new high-energy high-resolution pion beam with appropriate detector facilities is needed. In order to achieve this goal, work must be started immediately. 


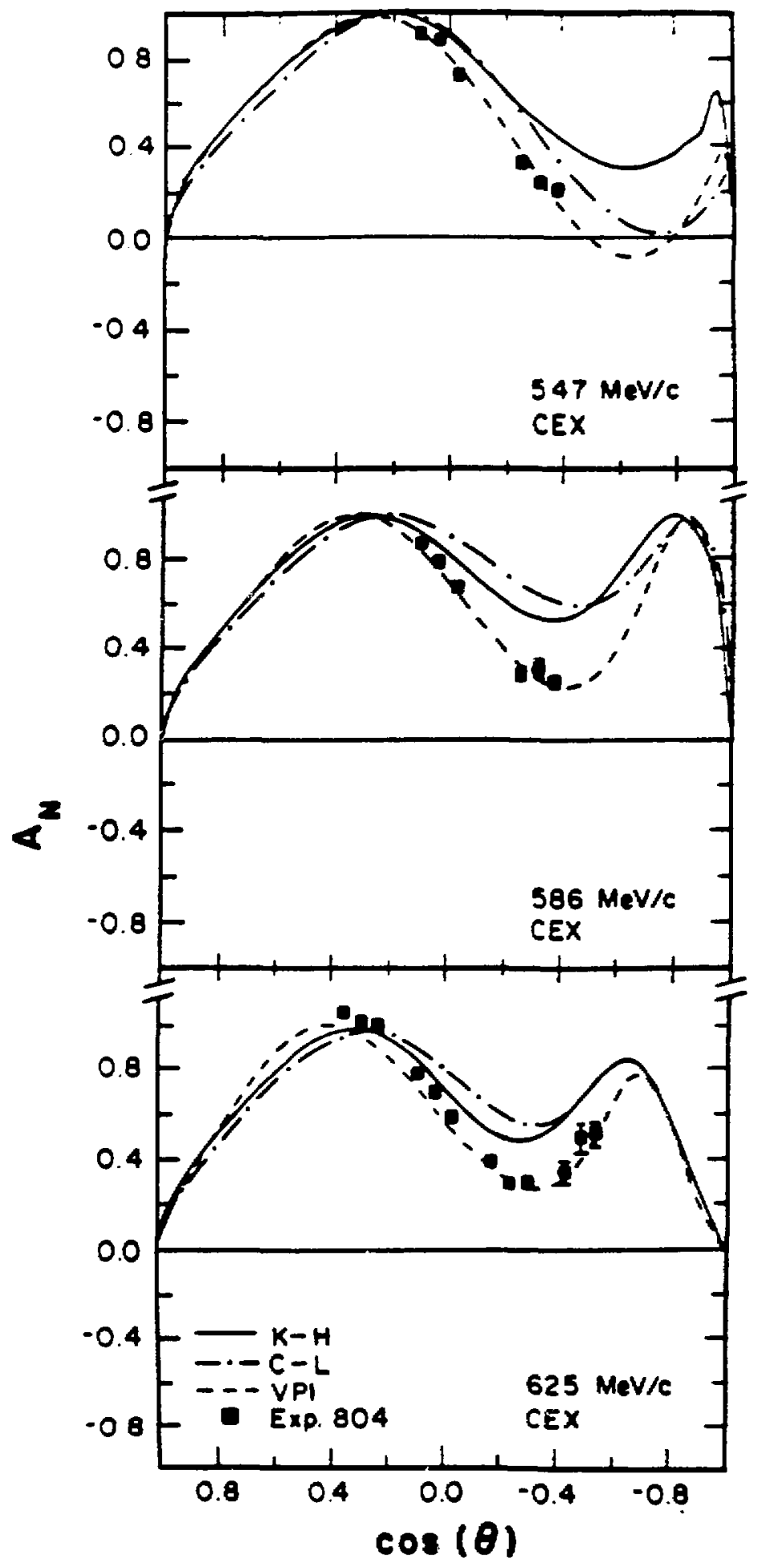

Fig. 1. Polarization asymmetry in the reaction $\pi^{-} p \rightarrow \pi^{0} n$. The curves represent predictions of phase-shift analyses. The solid curve is that of Karlsruhe-Heisinki, the dot-dashed curve is from Cutkosky-Livermore and the dashed curve is that of Arndt et al. (VPI). The latter contains (among other differences) two poles in the region of the Roper resonance. 


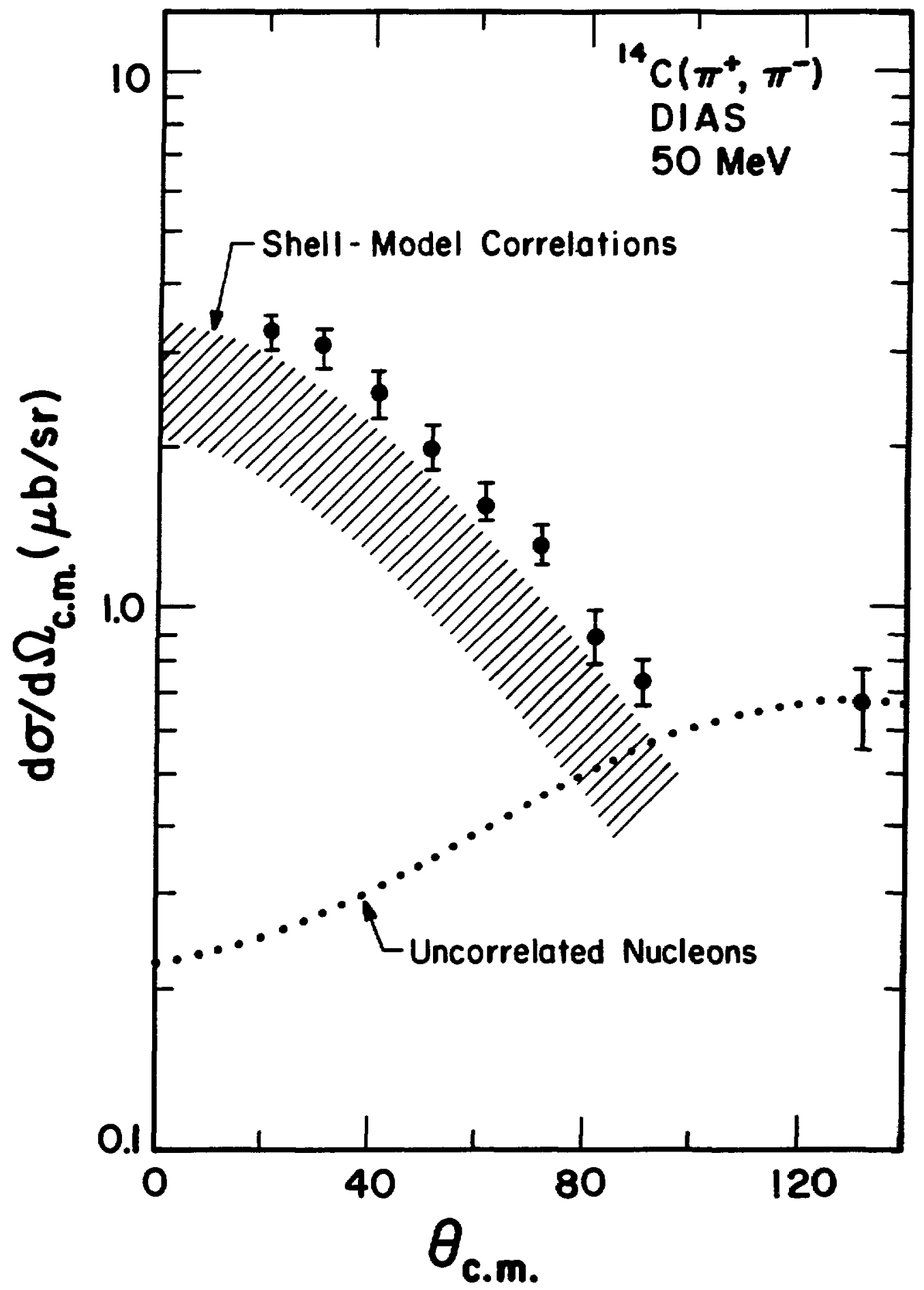

Fig. 2. Low-energy pion double charge exchange on ${ }^{14} \mathrm{C}$ to the analog state at $50 \mathrm{MeV}$. The dotted curve shows the result of a calculation based on the assumption that the two valence nucleons are uncorrelated. The band represents the approximate range of calculations based on wave functions with simple shell-model correlations. 


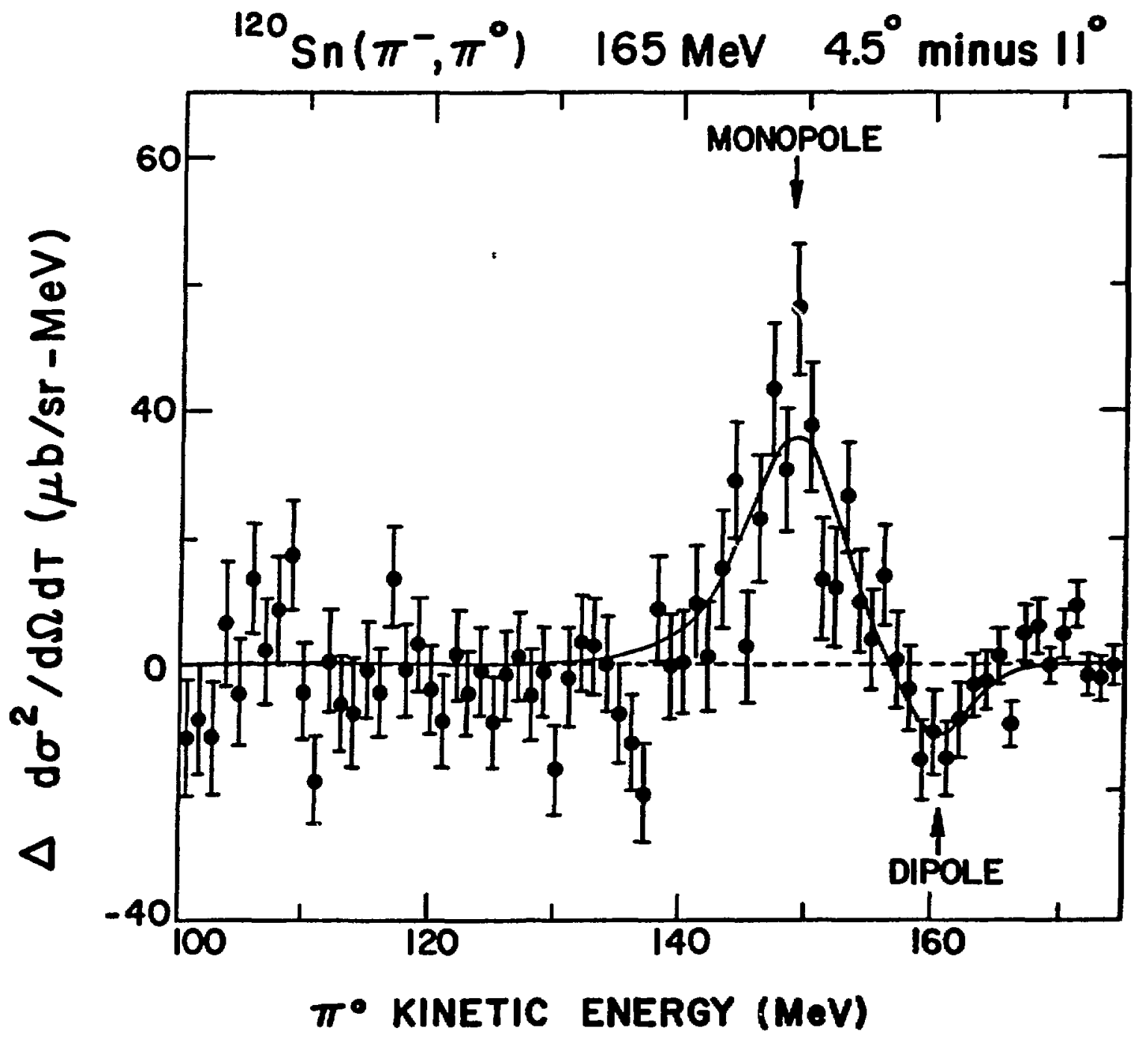

Fig. 3. Differences in the $4.5^{\circ}$ and $11.0^{\circ}$ double differential cross section for the reaction ${ }^{120} \mathrm{Sn}\left(\pi^{-}, \pi^{0}\right)$ at $T_{\pi}=165 \mathrm{MeV}$ as a function of the kinetic energy of the outgoing $\pi^{0}$. The positive excursion is the isovector monopole resonance, and the dip is the well-known giant dipole resonance. 


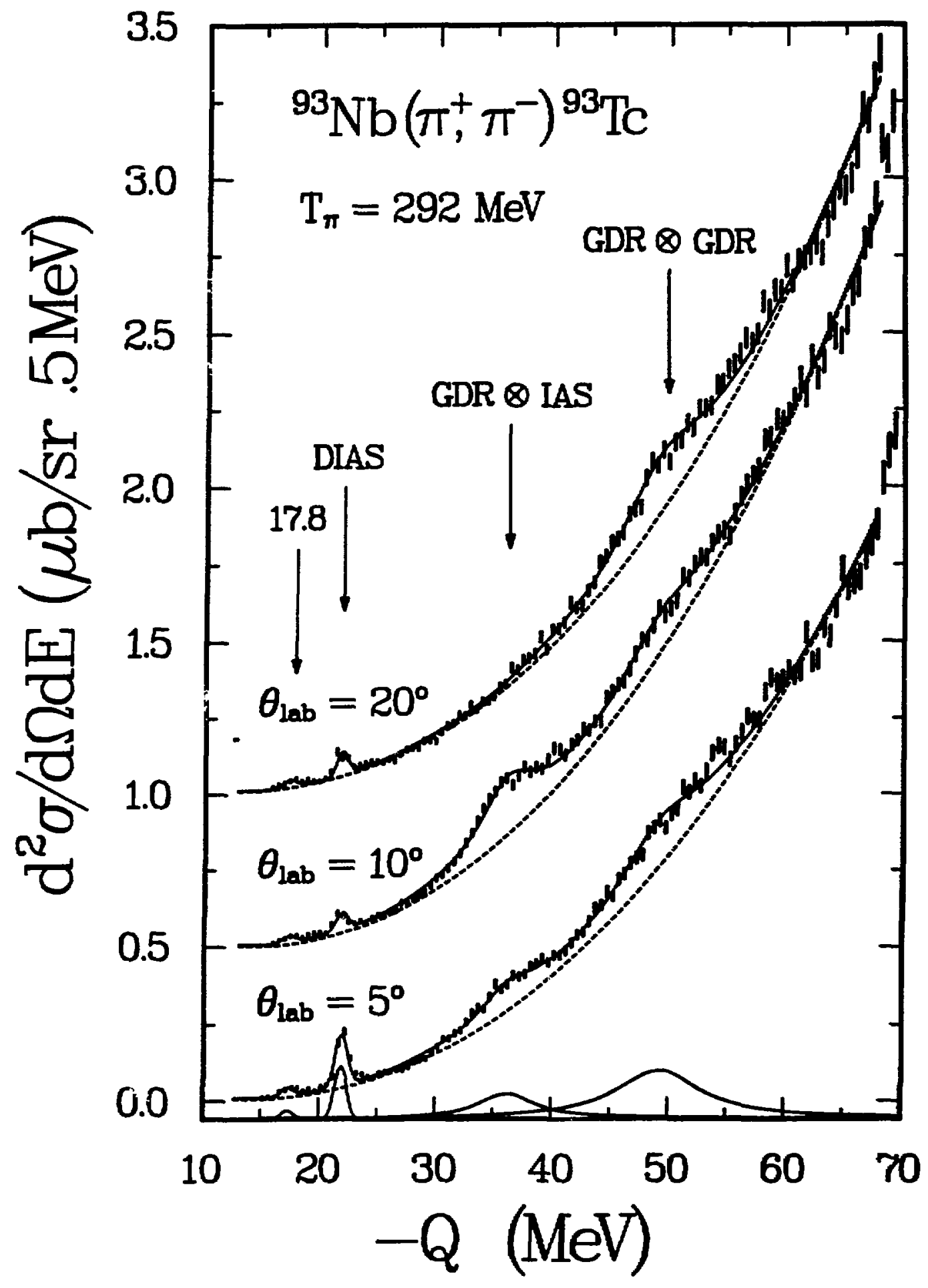

Fig. 4. Double differential cross-section spectra for the $\left(\pi^{+}, \pi^{-}\right)$reaction on a ${ }^{93} \mathrm{Nb}$ target at $T_{\pi}$ $=292 \mathrm{MeV}$ and $\theta_{1 \mathrm{ab}}=5^{\circ}, 10^{\circ}$, and $20^{\circ}$. The arrows indicate three double resonances observable in pion DCX: the DIAS, the GDR $\otimes$ IAS, and the GDR $\otimes$ GDR. 


\section{APPENDIX (TO THIS EXCERPT)}

Here we present a more complete list of accomplishments in pion physics in general over the past five years.

\section{PION-NUCLEON PHYSICS:}

Measurements of pion-nucleon elastic and charge-exchange scattering at low energies have been carried out which bear directly on the question of chiral symmetry breaking and the magnitude of the sigma term. The results suggest the possibility of a substantial sea of strange quark-antiquark pairs in the nucleon.

Measurements of pion charge exchange and single gamma production on polarized proton targets at higher energies have shown that phase-shift analyses that include a double-pole structure of the $N^{*}(1440)$ are preferred over others, and that the hypothesis that this resonance, if it is single, would correspond to the first excited state of the gluonic field is excluded.

\section{PION INTERACTIONS IN NUCLEI:}

A reliable description of pion scattering over the full range of energies investigated so far has been achieved through a refinement of the optical model.

An apparent transparency of the nucleus to $50-65-\mathrm{MeV}$ pions has been seen in measurements of single-charge-exchange scattering to analog states and of inelastic scattering to the first $\mathrm{0}^{+}$state in ${ }^{12} \mathrm{C}$.

Studies of double charge exchange to analog states with nuclei in the $f_{(z / 2)}$ shell have shown that it is possible to describe the process with two amplitudes that correspond to short- and longrange interactions between the two nucleons involved.

The range of energy available for high-resolution studies of pion-nucleus interactions has been extended above $500 \mathrm{MeV}$, and initial measurements of elastic and double-charge-exchange scattering have been carried out. Results in double charge exchange indicate possible disagreement with the strengths of ground-state transitions as predicted by the two-amplitude model above.

Unexpectedly large cross sections for double-charge-exchange scattering to nonanalog states have been found, both at low and resonance energies. Those at resonance energies follow the behavior expected from Delta production in the nucleus, and those at low energy follow generally the same behavior as analog transitions.

Coincidence studies of $(\pi, \pi p)$ quasifree scattering at resonance energies in both the elastic and single-charge-exchange channels have found strong evidence for Delta production followed by Delta-nucleon knockout as an important reaction mechanism.

Measurements of inclusive double charge exchange in the resonance region have found an unexpected double-peak distribution for light nuclei and an unexpected saturation of the total cross section for $\left(\pi^{-}, \pi^{+}\right)$in heavy nuclei. These results are consistent with a mechanism that is dominated by sequential single-charge-exchange scattering. 
Measurements of pion absorption at rest and in flight have established mechanisms involving more than two nucleons and have shown that the two-nucleon absorption probability is much greater with isospin-zero pairs than with isospin-one pairs.

The initial observation of pion-induced production of eta mesons to a definite final nuclear state was made.

Measurements of inclusive production of eta mesons on a range of nuclear targets for the determination of the eta-nucleon reaction cross section were made. This cross section is in substantial agreement with the additive quark model.

\section{NUCLEAR STRUCTURE:}

Studies of double charge exchange with heavy nuclei have produced the first observations of double isobaric analog states in such nuclei, and in some cases information on the widths of these states has been extracted.

Comparisons of $\pi^{+}$and $\pi^{-}$inelastic scattering on several nuclei have led to the extraction of many transition matrix elements. The agreement of the results with those obtained by electromagnetic methods is excellent.

The first evidence for the expected giant monopole resonance in nuclei was found in chargeexchange scattering at the forward direction on a range of nuclei.

Giant dipole resonances built on isobaric analog states and on the giant dipole resonance itself have been seen for the first time in double-charge-exchange scattering.

The strength of the Lambda-nucleon spin-orbit interaction has been determined to be small compared to that of the nucleon-nucleon spin-orbit interaction.

The initial observation of a series of particle-hole states deeply embedded within a heavy nucleus has been made in hypernuclear studies. 


\title{
APPENDIX \\ MESON SPECTROSCOPY WITH LIGHT HADRONS
}

\author{
J. R. Comfort \\ Arizona State University \\ T. Goldman \\ Los Alamos National Laboratory
}

\begin{abstract}
The spectroscopy of mesons is a subject that is still not well understood in spite of their importance as elementary particles and as mediators of the nuclear interaction. Frontier models of the QCD structure of mesons and baryons in terms of quarks and gluons could be tested if there were better identification of the mesons in terms of their multiplets or families and more complete knowledge of their decays and other properties. New insights can be achieved by the use of light hadronic probes such as pions and protons in conjunction with complementary studies with electromagnetic probes. The studies should be of high quality and will require large beam fluxes, precision detectors of large solid angle, and should make use of polarization observables wherever possible.
\end{abstract}

\section{Introduction}

Conventionally, mesons are the mediators of the nuclear interaction, with effective ranges inversely proportional to their masses. Studies with the lowest-mass meson, the pion, continue to have very high priority in view of the availability of high quality beams and its dominant role in nucleon-nucleon interaction and nuclear structure.

Heavier mesons are also very important, particularly for the shorter-range portions of the strong interaction that are less well understood. Although a $\sigma(\epsilon)$ meson has never been firmly identified, possibly due to its expected width of several hundred $\mathrm{MeV}$ with a mass near $600 \mathrm{MeV}$, it has long played an important role in nuclear-structure theories. For example, the currently popular Dirac pheonomenological treatment of nucleon-nucleus interactions, and the quantum hydrodynamical description of nuclear structure, have their foundations in $\operatorname{scalar}(\sigma)$ and vector $(\omega)$ mesons.

The isovector $\rho$ meson is also frequently incorporated in theoretical treatments of pion-nucleon and pion-nucleus interactions. For example, it plays a crucial role in the spin-isospin response of the nuclear medium near the threshold to pion condensation. Model calculations are very sensitive to the $\rho N N$ coupling. This coupling is determined only indirectly and is uncertain by as much as a factor of two.

Although many other issues could be cited, it is clear that a full understanding of the strong interaction between elementary particles both inside and outside of the nuclear medium cannot be achieved without a thorough understanding of the mesons. The very interesting short-range portions, which are vital for understanding the role and significance of two-nucleon correlations in the nuclear medium, require a more complete study of the heavier mesons and their interactions with nucleons and with each other. 


\section{QCD Models}

Quarks and gluons are playing an increasingly important role in discussions of nuclear interactions and structure. Quite a few experiments have been proposed or pursued in the hopes that subnucleonic degrees of freedom might be observed in nuclei, most without distinguishing success. The mesonic description of the $N \boldsymbol{N}$ interaction appears to be viable for most descriptions of observed phenomena.

Nevertheless, quarks and gluons are central to current descriptions of the elementary particles. The primary theory of the strong interaction, $Q C D$, successfully describes behaviors at high energies. However, the magnitude of its coupling constants precludes its direct application in the low-energy regime except for features at large momentum transfer that can be calculated perturbatively. Instead, several models of QCD have been developed for low-energy descriptions, including the spectra of mesons and baryons as well as their properties.

Several of the QCD models are able to calculate many of the properties of the mesons well. Many of the models also predict the existence of exotic mesons such as those of $q^{2} \bar{q}^{2}$ structure. The corresponce between the spectrum of known (or possibly known) mesons and the calculated states is often very poor. The problems arise in large part from experimental limitations and uncertainties in the analyses whereby even the masses and widths of most of the higher-lying mesons are quite uncertain and reported values tend to change from year to year.

Experimental facilities have developed to a point that programmatic studies can now be attempted with new abilities and greater precision. Studies of meson and baryon spectroscopy are expected to be a significant part of the CEBAF experimental program. Complementary studies with hadrons are strongly needed in order to establish a complete picture. The study of the spectroscopy and structure of mesons is analogous to the study of nuclei, where quarks and gluons rather than nucleons and photons are the principal ingredients.

\section{Meson Families}

The lowest-lying multiplets of normal $q \bar{q}$ mesons form nonets (an octet and a singlet) consisting of two $I=0$ particles, a triplet of $I=1$ particles, and four strange $I=1 / 2$ mesons (including antiparticles). Some of the families are well established. Thus, the pseudoscalar nonet consists of the $\pi(139 ; I=1), \eta(549 ; I=0), \eta^{\prime}(958 ; I=0)$, and $K(494,498 ; I=1 / 2)$ mesons; and the vector nonet consists of the $\rho(770 ; I=1), \omega(782 ; I=0), \phi(1020 ; I=0)$, and $K^{*}(892,896 ; I=1 / 2)$ mesons.

The spectrum of the scalar mesons is not well agreed on. The Particle Data Group identifies the nonet as the $a_{0}(980 ; I=1)$ [originally $\delta$ ], $f_{0}(975 ; I=0)$ [originally $S^{*}$ ], $f_{0}(1400 ; I=0)$ [originally $\epsilon(1300)$ ], and $K_{0}^{*}(1430 ; I=1 / 2)$ [originally $\kappa(1350)$ ]. This scheme has no room for a scalar in the region of $600 \mathrm{MeV}$, as used in models of nuclear interactions applied to nuclei.

Alternative schemes have been proposed. Within the framework of a QCD model that successfully describes the pseudoscalar and vector mesons in terms of a $q \bar{q}$ structure ${ }^{1}$ Jaffe predicted a low-lying scalar $q^{2} \bar{q}^{2}$ multiplet consisting of an $\epsilon(\sim 700 ; I=0), \delta(975 ; I=1), S^{*}(980 ; I=0)$, and a $\kappa(\sim 900 ; I=1 / 2)$. The $q \bar{q}$ multiplet is predicted to lie at higher masses. For different reasons related to the spontaneous breakdown of chiral symmetry, Scadron obtains a virtually identical 
spectrum for a $q \bar{q}$ structure in a relative $L=1$ state. ${ }^{2}$ Data considered by Estabrooks favor the existence of an $\epsilon$ near $800 \mathrm{MeV}$, but the $\kappa$ is suifgested at around $1540 \mathrm{MeV} .^{3}$

Further insight into the issue of the scalars is provided by recent work that involved polarization observables. In an analysis of data from the $\pi^{+} \vec{n} \rightarrow \pi^{+} \pi^{-} p$ reaction at 6 and $12 \mathrm{GeV} / c$, taken on a polarized deuteron target, ${ }^{4}$ Svec suggested that the polarization observables could be used to project out the possible existence of a scalar meson (the $\kappa$ ?) near $750 \mathrm{MeV}{ }^{5}$ It should be noted that this possible meson is on top of the $\rho$ and $\omega$ resonances and would not be identifiable at all without some filter such as polarization observables.

\section{Meson Production by Inclusive and Exclusive Reactions}

One can readily consider a program for the production of mesons by means of inclusive and exclusive reactions initiated by pions and protons. The program is analogous to that which is studied at electron accelerators (such as the future program at CEBAF) with $\left(\gamma\right.$, meson) or $\left(\epsilon, e^{\prime}\right.$ meson) reactions.

In terms of the relativistic invariants of energy loss $\omega$ and four-momentum transfer $t$, the $\left(e, e^{\prime}\right)$ reaction on a proton at $4 \mathrm{GeV}$ can span the $\omega$ - $t$ plane for $-t$ ranging from 0 to about $6.5 \mathrm{GeV}^{2}$ when $\omega$ ranges from 0 to $4 \mathrm{GeV}$. Mesons can be produced for masses up to and beyond $2 \mathrm{GeV}$. At $6 \mathrm{GeV}$, which CEBAF might eventually achieve, $-t$ can extend up to more than $10 \mathrm{GeV}^{2}$ while $\omega$ ranges up to $6 \mathrm{GeV}$. The range of $t$ is reduced as the meson mass increases. The $(\gamma$, meson $)$ reaction is sensitive only to the kinematic line that corresponds approximately to electron elastic scattering from a proton. The range along the line is limited by the beam energy and the meson mass. It should be realized, of course, that the production of virtual photons in the $\left(e, \epsilon^{\prime}\right)$ reaction decreases rapidly with four-momentum transfer, approximately as $1 / t^{2}$.

Kinematics for the pion $\left(\pi, \pi^{\prime}\right.$ meson) and ( $\pi$, meson), and the proton $\left(p, p^{\prime}\right.$ meson) reactions are similar to those for electrons. The range of $\omega$ and $-t$ is extended slightly and the reactions take place at more forward angles for the same point in the $\omega$ - $t$ plane. Again, the $(\pi$, meson) reaction gives a single kinematic line corresponding approximately to pion elastic scattering. Hence, the same kinematic regions in the $\omega$ - $t$ plane can be mapped out by electron-, pion-, and proton-induced reactions. The different features of the probes can be used to compare and contrast the results of the experiments, and to interpret the data in terms of the existence of mesons, their structural properties, and their interactions with other hadrons.

The pion and proton energies required for such a program are typically at least $2 \mathrm{GeV}$. For pions near $1-1.2 \mathrm{GeV}$, for example, only $\pi$ and $\eta$ mesons can be produced by the ( $\left.\pi, \pi^{\prime}\right)$ inclusive reaction, while the $(\pi, \rho)$ reaction is just barely above threshold. A dedicated study of the spectrum of scalar mesons should include searches for a strange scalar meson $(\kappa)$ from well below $1 \mathrm{GeV}$ to perhaps as high as $1.6 \mathrm{GeV}$. Since $\kappa$ production also requires the excitation of the proton to a $\boldsymbol{\Lambda}$ or $\Sigma$, the kinematic thresholds are well above $2.5 \mathrm{GeV}$. Such searches are vital to understanding current conflicting models of the scalar mesons, as well as the hope of locating the $\sigma$ ( $\sigma$ ) near $600 \mathrm{MeV}$ that is so important to nuclear physics. 


\section{Needed Facilities}

The physics discussed here will require new facilities. Since a large portion of the $\omega$-t plane needs to be explored (in part to provide good data for extrapolations of the interactions to the pole positions of the mesons), a high beam flux will be required for studies at large $|t|$. A large-solidangle, multiparticle detector (approximately $4 \pi$ ) will be needed as well. It should have the ability to handle polarized targets in order to make use of polarization observables.

A companion to the program discussed here is the study of meson production in nuclei. Such a program is vital in order to understand the possible modification of the elementary interactions within the nuclear medium. An example is the $\rho N N$ interaction. It has been suggested recently that the rho-nucleon interaction is describable by effective masses that are density dependent. ${ }^{6}$ This feature was shown to account for discrepancies that had been observed for $K^{+}$scattering from ${ }^{12} \mathrm{C}$, which were originally accounted for by a quark-based nucleon-swelling model. ${ }^{7}$

Whether or not density-dependent interactions are a valid or fruitful description, it is clear that a detailed understanding of mesons is essential to a full treatment of hadronic interactions in nuclei.

\section{References}

1. R. L. Jaffe, Phys. Rev. D15, 267 (1977).

2. M. D. Scadron, Phys. Rev. D 26, 239 (1982).

3. P. Estabrooks, Phys. Rev. D 19, 2678 (1979).

4. A. de Lesquen et al., Phys. Rev. D32, 21 (1985).

5. M. Svec, AIP Conf. Proc. 132, 68 (1985).

6. G. E. Brown et al., Phys. Rev. Lett. 60, 2723 (1988).

7. P. B. Siegel, W. B. Kaufmann, and W. R. Gibbs, Phys. Rev. C31, 2184 (1985). 